\title{
Adaptive resource allocation for cognitive radio networks with multiple primary networks
}

\author{
Ye Wang, Qinyu Zhang*, Yalin Zhang and Peipei Chen
}

\begin{abstract}
In this article, adaptive resource allocation (ARA) is investigated for multiple primary networks based-cognitive radio networks under a more practical system model, where the bandwidth of each secondary user is assumed to be limited and the maximum allowable interference for each primary network is different. We first formulate the ARA as a constrained optimization problem with the objective function of maximizing the proportional fairness-based ergodic sum capacity. The multiple constraints optimization problem is NP-hard and therefore, we propose a scheme to decompose the optimization problem into two unconstrained optimization problems by designing alternative objective functions and penalty functions. Then, a suboptimal heuristic solution framework based on particle swarm optimization is proposed to solve the unconstrained optimization problems. Computation simulations are carried out and the results show that the proposed scheme outperforms traditional ARA schemes.
\end{abstract}

Keywords: Cognitive radio networks, Resource allocation, Multiple primary networks, Particle swarm optimization

\section{Introduction}

Recently, cognitive radio networks (CRNs) [1] get extensive attentions to alleviate the contradiction between the scarcity and low-utilization of the spectrum resources. In CRNs, two approaches are widely studied for dynamic spectrum access (DSA), termed as underlay mode and overlay mode. In underlay mode, secondary users (SU) transmit over all the frequency bands as long as the serviced SUs do not cause excessive interference to primary users (PU). One possible drawback of this mode is that it requires exact information of all primary receivers' positions, which usually expenses a high complexity of hardware and computation. On the other hand, the basic idea of overlay is to detect the absence/presence of licensed primary radios, and opportunistically use the idle band for transmissions without causing harmful interference to the authorized signals $[2,3]$. Compared to the underlay mode, the overlay mode is more practical and easily accepted by primary systems. Also, the overlay spectrum access can further improve spectrum efficiency by exploiting multiuser diversity and adaptive resource allocation (ARA)

*Correspondence: zqy@hit.edu.cn

Department of Electronic and Information Engineering, Shenzhen Graduate School, Harbin Institute of Technology, Shenzhen, China algorithm. In this article, we consider the problem of ARA for overlay CRNs.

ARA is one of the most effective methods to improve the spectral efficiency and has widely been investigated in the last decade. Unlike existing wireless communication networks, the ARA problem in CRNs is more challenging. First, the detection errors of spectrum sensing greatly deteriorates the performance of ARA algorithm $[4,5]$. The authors of [4] proposed an iterative algorithm to obtain the optimal sensing time and the corresponding power allocation strategy with imperfect sensing information. The authors of [5] presented a primal-dual decomposition-based cross-layer scheduling for power allocation and subchannel assignment with raw sensing information. Furthermore, cognitive radios are usually required to be self-regulating, to control the interference to primary systems in a tolerable level [5-7]. The authors of [5] considered the average interference constraint on the nearest PU receiver. In [6], a total interference constraint to a certain PU who uses the same channel with SU is imposed. In $[8,9]$, the interference control is considered as a total power constraint of all SUs.

Besides, because of the absence of the license, the available spectrum resources for SUs are usually temporary and unstable, which results in transmission interruption with high probability. One of considerable solutions is

\section{至 Springer}

(c) 2012 Ye et al: licensee Springer. This is an Open Access article distributed under the terms of the Creative Commons

Attribution License (http://creativecommons.org/licenses/by/2.0), which permits unrestricted use, distribution, and reproduction in any medium, provided the original work is properly cited. 
to enable SUs to search for spectrum resources from multiple primary networks (PRNs) simultaneously. The authors of [10] considered the mobile users operating on different radio access technologies (RATs), and the single-user case is analyzed. In the multiple PRNs, however, the available spectrum resources are usually quite wide as well as discontinuous. This leads to that the cognitive radios operating on such spectrum resources are price prohibitive due to the increase of sampling rate. In addition, different PRNs restrict different power values of CRNs in one resource allocation, which greatly increases the computational complexity of ARA.

This article aims to design an effective ARA algorithm in multiple PRNs environment. Specifically, we assume that the spectrum resources available for SUs are distributed in multiple PRNs, and discontinuous in frequency domain. Furthermore, the sampling rates of SUs are limited for practical consideration. Other constraints concerned in traditional multiuser communications networks like proportional fairness and bit error rate (BER) are also involved in this study.

We formulate these concerns as a constrained optimization problem with the objective function of maximizing sum capacity. The formulation is a mixed integer nonlinear programming (MINP) that is a typical NP-hard problem. Therefore, the most intractable problem is to find a suboptimal alternative with polynomial time complexity. In this article, we proposed an integral solution to the optimization problem. The main works and contributions of this article can be summarized as follows.

(1) The ARA problem for CRN in multiple PRNs is formulated as a constrained optimization problem.

(2) A hybrid alternative objective function instead of sum capacity is proposed to balance the tradeoff between the complexity of optimization and the speed of convergence.

(3) The optimization problem is transformed into two unconstrained problems through the design of penalty functions. In particular, we propose multiplicative penalty functions that are independent with channel gains and power limits, contributing to the algorithm more robust.

(4) A particle swarm optimization (PSO)-based heuristic intelligence algorithm is proposed to solve the two unconstrained problems. Specifically, we first present an initial topology of PSO according to the fact that an SU suffers channel fading of the same level for all frequencies (because large scale attenuation plays the key role for wireless channel). The proposed topology can greatly decrease the computation complexity from $O\left(K^{N}\right)$ to $O\left(K^{3}\right)$. Based on this, we design different particle structures for subchannels and power allocation, respectively.
In order to verify the proposed ARA algorithm, three computer simulations are carried out. The first one illustrates the efficiency of the proposed hybrid objective function in terms of convergence and quality; for comparison, we put a classical ARA algorithm [11] into the same scenario. The simulation results reflect that the proposed outperforms the algorithm in [11] both in capacity and proportional fairness; at last, we discuss the effect of imperfect sensing information, and a possible optimization of sensing strategy is proposed.

The rest of this article is organized as follows. The following Section "Related studies" introduces the state of the art in ARA for CRNs. In Section "System model and problem formulation", we describe the system model and formulate the ARA as a constrained optimization problem. In Section "Objective function and problem decomposition", we decompose the optimization into two unconstrained optimization problems. In Section "Particle swarm optimization based framework", we discuss the solution to the optimization problems by a PSO based algorithm in detail. The computational complexity of proposed is analyzed in Section "Computational complexity analysis". To verify the proposed scheme, computer simulations are carried out in Section "Results and discussion", and the brief conclusions are drawn at last.

\section{Related studies}

The main foundation of ARA is based on that different users suffer from different wireless channel fading. ARA algorithms can adaptively allocate a dimension to the users, and achieve higher capacity. Two classes of resource allocation techniques have widely been addressed in traditional OFDM-based wireless communication systems, namely, (1) margin adaptive (MA) [9] and (2) rate adaptive (RA) [12]. The MA aims to minimize the overall transmit power, given the constraints on the users transmit rates or BER. The RA aims to maximize users' capacity sum, given a total power constraint. Both optimization problems are MINP problem, and computationally prohibitive. The authors of [13] presented a suboptimal resource allocation algorithm by transforming the MA problem into a convex optimization problem. The authors of $[11,14]$ discussed RA problem with the constraint of proportional rate, and proposed an optimal power allocation scheme by the Newton-Raphson method.

Right now, a lot of research works is currently ongoing to deal with the optimization of the spectrum utilization in CRNs. The authors of [5] presented a primal-dual decomposition approach for the RA problem with raw sensing information. However, they ignored proportional rate and fairness, which ensure that all users suffering different channel fading can experience the similar quality of service $[15,16]$. In this article, we assure proportional fairness among SUs by imposing a set of nonlinear constraints 
into the optimization problem and formulate a new optimization problem. Regarding to interference control, an important concept named "interference temperature" [17] is widely used to evaluate the interference on the primary system. Wang et al. [5] considered the average interference constraint on the nearest PU receiver. Nguyen and Lee [6] imposed a total interference constraint on PU, who uses the same channel with SU. Although "interference temperature" is an exact measurement, it is difficult to implement in realistic systems. To calculate the aggregation interference on each PU, the sensing algorithm based on receivers is needed, which usually requires information of receivers' positions and channel gains from each SU transmitter to the corresponding PU receiver. In this article, we simplify the constraint with an only total power limit on specific PRN. This simplification is reasonable since the value of power constraint could be negotiated between PRN and CRN through a spectrum broker [18]. Choi et al. [10] considered the mobile users who can access different RATs, and the single-user case was analyzed. In this article, we equip the $\mathrm{SU}$ as the same capability of operating on crossing bandwidth and extend the scenario to multiuser case. Further, we consider that the sampling capability of SU is limited and only part of the spectrum resources can be exploited at any time.
Because of extra constraints in CRNs, it is nontrivial to obtain optimal solution for ARA problem. Bio-inspired swarm intelligence-based optimization algorithms $[19,20]$, as an effective alternative solution, have widely been adopted in CRNs. A successful example of exploring the swarm intelligence algorithms in CRN is the testbed designed by Virginia Tech group [21,22], which shown the flexibility and stability of CRN with the genetic algorithm (GA)-embedded cognitive engine. Newman et al. [23] proposed a GA-based cognitive radio suitable for Emergency (minimize BER) and Low Power (minimize power consumption, like MA in a traditional network scenarios). Since then, lots of alternative schemes such as the quantum genetic algorithm [24], cross entropy [25], and PSO [26] is exploited to address the problems of resource allocation. PSO is proposed as a new swarm intelligence algorithm in recent years due to its capabilities of convergence rapidity, optima finding, and matching problem easily. In this article, we consider PSO as a tool to solve the problem of resource allocation in CRNs.

\section{System model and problem formulation \\ System model}

We consider an infrastructure-based CRN system under multiple PRNs, as depicted in Figure 1. PRN $i$ is licensed $N_{i}$ nonoverlapping orthogonal frequency subchannels.

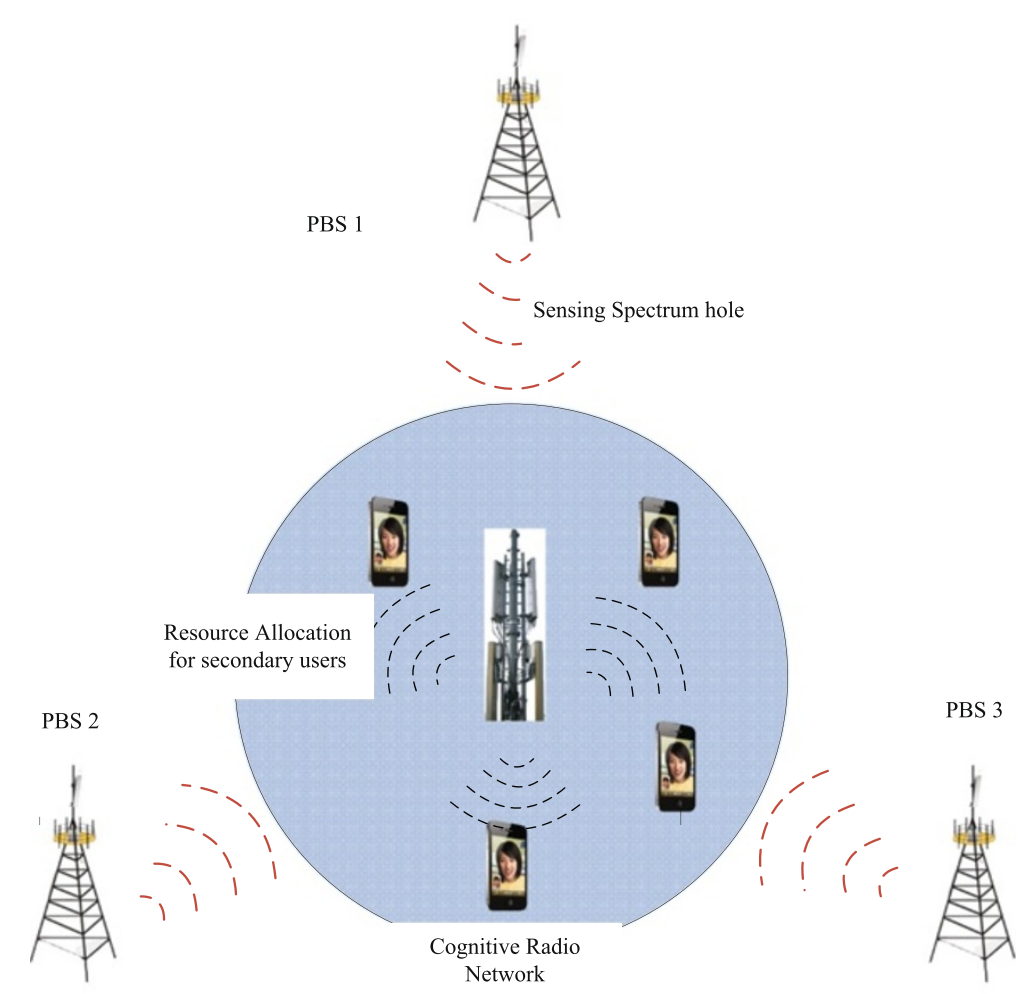

Figure 1 The cognitive OFDM system with multiple primary networks. 
The bandwidth of each subchannel is $W \mathrm{~Hz}$. For simplicity, we assume that there is no guard band and then the spectrum spacing between two adjacent subchannels is also $W \mathrm{~Hz}$. The coverage radius of cognitive base station is $d$ and the distance between each primary base station (PBS) and cognitive base station (CBS) is $2 d$. Within one resource allocation, there exist $K$ active SUs in the CRN.

Before performing resource allocation, the CBS first senses the primary signals from its neighboring PBSs. According to the sensing results, the CBS decides whether the corresponding spectrum band is busy or idle. Then, based on the spectrum states, the CBS dynamically allocates the available spectrum resources to SUs in service. In practice, the spectrum sensing is not always perfect and accurate. Two sensing errors, miss detection (MD) and false alarm (FA) are inevitable for spectrum sensing. An MD means that an SU identifies a channel unoccupied while in fact a PU is transmitting on which. An FA means that an SU identify a channel BUSY while that is actually IDLE. We denote the FA probability and MD probability as $P_{f}$ and $P_{m}$, respectively. Both of them are the signal-tonoise ratio (SNR) as well as sensing algorithm dependent. If there are $N_{i}^{\text {busy }}$ busy subchannels and $N_{i}^{\text {idle }}$ idle subchannels in PRN $i$, the number of available subchannels for CRN is

$$
N_{i}^{\text {sense }}=\left(1-P_{f}\right) N_{i}^{\text {idle }}+P_{m} N_{i}^{\text {busy }}
$$

Hence, the total available subchannels for CRN is $N^{\text {sense }}=\sum_{i=1}^{S} N_{i}^{\text {sense }}$. We assume that the resource allocation period is updated as fast as CSI feedback between CBS and SUs, and perfect instantaneous CSI is assumed available for the CBS. It is also assumed that information of both resource allocation results and CSI are transmitted through a cognitive pilot channel [27].

\section{Problem formulation}

The objective in this study is to find a resource allocation solution to maximize the total ergodic capacity of CRN. We denote $R_{k}^{i}$ as the ergodic capacity of user $k$ in PRN $i$, which can be written as

$$
\begin{aligned}
R_{k}^{i}= & \sum_{m=1}^{\left(1-P_{f}\right) N_{i}^{\text {idle }}}\left(\frac{\rho_{k, m}^{i}}{\left(1-P_{f}\right) N_{i}^{\text {idle }}} \log _{2}\left(1+\frac{p_{k, m}^{i}\left(h_{k, m}^{i}\right)^{2}}{\Gamma_{k} N_{0} W}\right)\right. \\
& +\sum_{m=1}^{\left(1-P_{d}\right) N_{i}^{\text {busy }}} \frac{\rho_{k, m}^{i}}{P_{m} N_{i}^{\text {busy }}} \log _{2} \\
& \left.\times\left(1+\frac{p_{k, m}^{i}\left(h_{k, m}^{i}\right)^{2}}{\Gamma_{k}\left(\sum_{i=1}^{S} I_{k, m}^{i}+N_{0} W\right)}\right)\right)
\end{aligned}
$$

where $N_{0}$ is the two-sided noise spectral density. We assume that the noise is additive white gaussian noise, and all spectrum has the same value of $N_{0} \cdot \Gamma_{k}=$ $\frac{N_{0}}{3} Q^{-1}\left(\frac{B E R}{4}\right)^{2}$ is the SINR gap due to modulation, and BER is the BER for SUs. $I_{k, m}^{i}$ represents the interference on subchannel $m$ of SU $k$, from the PRN $i . h_{k, m}^{i}$ is the channel gain for SU $k$ in subchannel $m$ of the PRN $i$ and $p_{k, m}^{i}$ is the power allocated in subchannel $m$ of the PRN $i, \rho_{k, m}^{i}$ can either be 1 or 0 , indicating whether subchannel $m$ of PRN $i$ is allocated to SU $k$ or not.

Hence, the total sum capacity for all SUs can be written as:

$$
R=\sum_{k=1}^{K} \sum_{i=1}^{S} R_{k}^{i}
$$

To avoid interference on PRNs or control interference at a tolerable level, a power control scheme is necessary in CRNs. Traditionally, the "interference temperature" is an evaluation indicator representing the aggregation interference of SUs in terms of the power spectrum [17]. In order to evaluate the interference temperature from $\mathrm{SU}$ transmitter to each PU receiver, we need the position information of PUs and the channel gains between the $\mathrm{SU}$ transmitter and all PU receivers. This requirement is too expensive for implementation. Therefore, in this article, we adopt a simple scheme which restrains the total power of CRN in each PRN [8,9]. Denote $P_{\text {total }}^{i}$ as the total available power in PRN $i$, and the power constraint can be written as:

$$
\sum_{m=1}^{N_{i}^{\text {sense }}} \sum_{k=1}^{K} p_{k, m}^{i} \leq P_{\text {total }}^{i}, \forall i
$$

where $p_{k, m}^{i}$ represents the power allocated to SU $k$ on subchannel $m$ of PRN $i$ and $P_{\text {total }}^{i}$ is the total power constraint for CRN in PRN $i$.

In addition, proportional fairness is also an important performance metric in multiuser wireless communication systems. Under the constraint of proportional fairness, we can explicitly control the capacity ratios among users, and generally ensure that each user can achieve its target data rate, especially for cell-edge users. In this article, we adopt the definition of proportional fairness index the same as in [14].

$$
\Phi\left(R_{1}, \ldots, R_{k} ; \gamma_{1}, \ldots, \gamma_{k}\right)=\frac{\left(\sum_{k=1}^{K}\left(R_{k} / \gamma_{k}\right)\right)^{2}}{K \sum_{k=1}^{K}\left(R_{k} / \gamma_{k}\right)^{2}}
$$

where $R_{k}=\sum_{i=1}^{S} R_{k}^{i}$ is the sum capacity of $\mathrm{SU} k$ in one allocation period and $\gamma_{k}$ is the expected transmit rate 
of SU $k$. The maximum value of 1 to be achieved when $R_{1} / \gamma_{1}=R_{k} / \gamma_{k}, \forall k$. To ensure each SU is served in one allocation period, we define a noncontinuous point $\Phi=0$, if $\forall R_{k}=0$.

Notably, to guarantee the total transmit rate, cognitive radios have to collect the spectrum resources from multiple PRNs. This leads that the available spectrum for CRN is usually band-crossing as well as discontinuous, as illustrated in Figure 2. If we assume that the cognitive radio can transmit over all spectrum at the same time, then the resource allocation is as trivial as that in a traditional network. However, this assumption seems too strong in practice. According to the theory of bandpass sampling, the sampling rate for bandpass signal of bandwidth $B$ must satisfy the following equation [28]:

$$
\frac{2 f_{c}+B}{m+1} \leq f_{s} \leq \frac{2 f_{c}-B}{m}
$$

where $m$ is an arbitrary integer, $f_{\mathrm{s}}$ is the sampling rate, $f_{\mathrm{c}}$ and $B$ are the central frequency and bandwidth of bandpass signal, respectively. That is to say, the maximal bandwidth a radio can operate on is subject to the $\mathrm{A} / \mathrm{D}$ capability, which is price prohibitive with the increase of $f_{\mathrm{s}}$. In this article, we consider SU is limited by bandpass capability, and denote $F_{k}^{\mathrm{lim}}$ as the width of maximal bandpass capability of SU $k$. Without loss of generality, we index the subchannel by lexicographical order, and denote $f_{n}$ as the central frequency of subchannel $n$

$$
f_{n}=\psi_{m}+\left(n-\sum_{i=1}^{m-1} N_{i}\right) W
$$

where the subchannel $n$ belongs to the PRN $m$ and $\sum_{i=1}^{m-1} N_{i}<n \leq \sum_{i=1}^{m} N_{i} . \psi_{m}$ is the starting frequency of PRN $m$. We denote $\Omega_{k}$ as the subchannels set allocated to SU $k$ in one allocation and define $\mathcal{F}_{k}^{\max }=\max _{n \in \Omega_{k}} f_{n}$ and $\mathcal{F}_{k}^{\min }=\min _{n \in \Omega_{k}} f_{n}$. The constraint of bandpass capability can be expressed as follows:

$$
\mathcal{F}_{k}^{\max }-\mathcal{F}_{k}^{\min } \leq F_{k}^{\lim }, \quad \forall k
$$

Mathematically, the optimization objective (3), and constraints (4), (5), (8) can be modeled as the following mixed-integer nonlinear programming:

$$
(\rho, \mathbf{p})=\arg \max R
$$

$$
\begin{aligned}
& \text { subject to }: C 1: p_{k, m}^{i} \geq 0, \forall k, m, i \\
& \qquad 2: \sum_{k=1}^{K} \sum_{m=1}^{N_{i}} p_{k, m}^{i} \leq P_{\text {total }}^{i}, \forall i \\
& C 3: \sum_{k=1}^{K} \rho_{k, n}=1, \rho_{k, n} \in\{0,1\}, \forall n \\
& C 4: \mathcal{F}_{k}^{\max }-\mathcal{F}_{k}^{\min } \leq F_{k}^{\lim }, \forall k \\
& C 5: \Phi=1
\end{aligned}
$$

where $\rho=\left(\rho_{1}, \rho_{2}, \ldots, \rho_{N^{\text {sense }}}\right)$ and $\mathbf{p}=\left(p_{1}, p_{2}, \ldots\right.$, $\left.p_{N^{\text {sense }}}\right)$ indicate the assignments of subchannel and power, respectively. The constraint $C 1$ implies all power value allocated to subchannels are positive; $C 2$ ensures the interference to PRNs at a tolerable level; $C 3$ indicates that each subchannel can only be used by one $\mathrm{SU}$ at any time; $C 4$ is the bandpass constraint of SU; $C 5$ is the proportional fairness constraint.

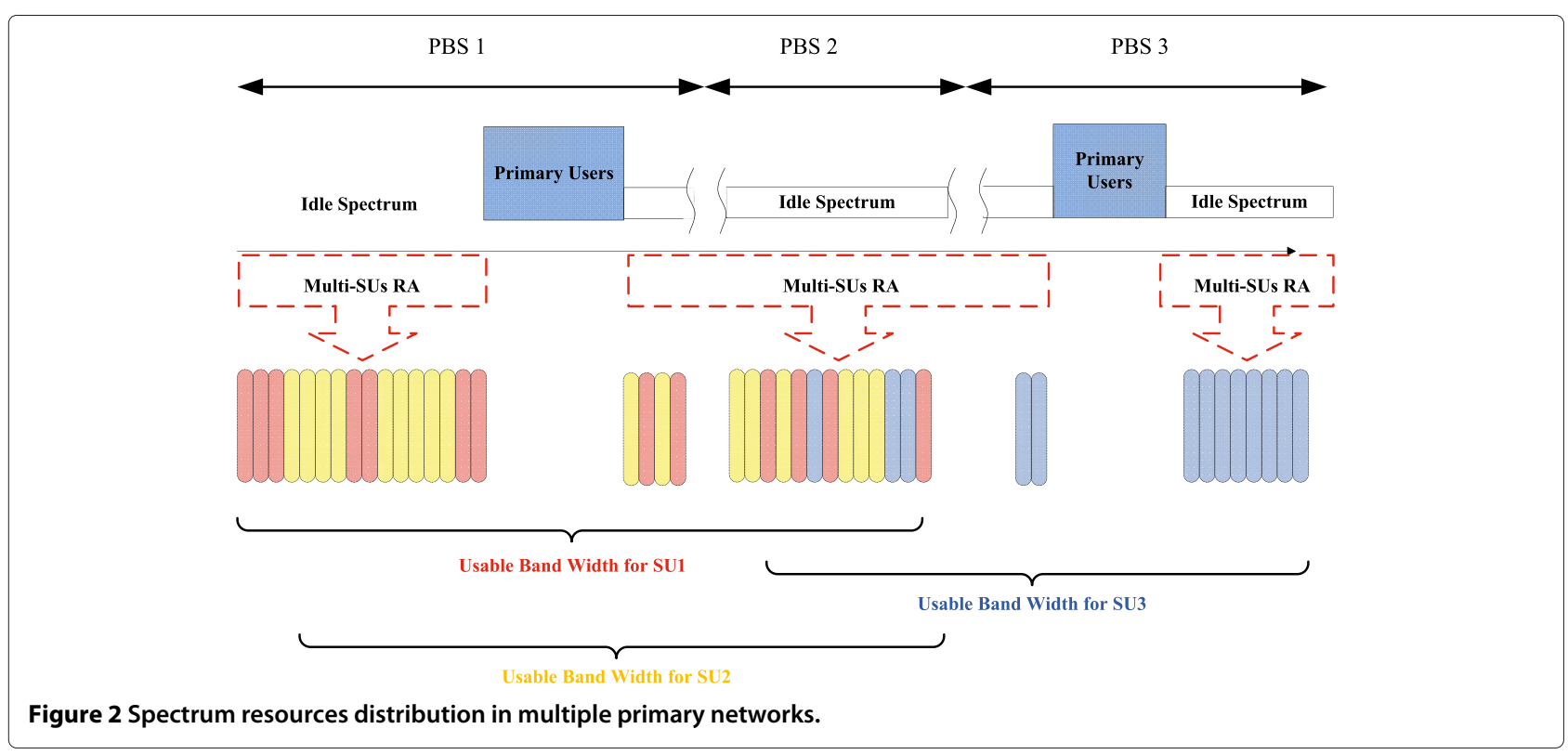




\section{Objective function and problem decomposition}

For a CRN system consisting of $N$ available subchannels and $K$ SUs that can crossover at most $m$ subchannels, there are at most $K^{N-m} C_{K-1}^{N-m}$ possible subchannel allocations. The feasible set is too huge to exhaust. Besides, the nonlinear constraints $C 4$ and $C 5$ in (9) increase the complexity to obtain the optimization solution. Therefore, it is prohibitive to find an optimizer in terms of computational complexity. In this article, we attempt to find a suboptimal alternative to decrease the complexity significantly while still delivering performance close to the global optimum.

Before designing of the suboptimal algorithm, we attempt to simplify the problem in (9) first. In Section "Objective function ", we analyze two equivalent objective functions to delete the constraint $C 5$, and then a hybrid objective functions is proposed. In Section "Problem decomposition", the problem in (9) is decomposed into subchannels allocation and power allocation, and each of them has only two constraints. Section "Penalty function" discusses the design of penalty functions, by which transforming the two sub-problems into unconstrained optimization problems.

\section{Objective function}

The global objective function is the maximum of ergodic sum capacity of SUs. It is difficult to achieve due to the constraint of proportional fairness. We need an equivalent objective function that is maximized simultaneously with global objective function. The maximum of proportional fairness and maximum of minimal capacity SU are two alternatives. For convenience, we denote the objective function of maximum of proportional fairness as MPF and maximum of minimal capacity SU as Max-Min-SU.

\section{MPF}

We relax the constraint of proportional fairness $\Phi \in(\epsilon, 1]$, where $\epsilon$ is a rational number close to 1 . Then we extract the constraint $C 5$ in (9) as an objective and remodel the problem as a multi-objective problem that:

$$
\begin{array}{r}
\left(\rho^{*}, \mathbf{p}^{*}\right)=\arg \left(\max \sum_{k=1}^{K} R_{k} \& \max \Phi\right) \\
\text { subject to : } C 1: p_{k, n}^{i} \geq 0, \forall k, n, i \\
C 2: \sum_{k=1}^{K} \sum_{m=1}^{N_{i}} p_{k, n}^{i} \leq P_{\text {total }}^{i} \forall i \\
C 3: \sum_{k=1}^{K} \rho_{k, n}^{i}=1, \rho_{k, n}^{i} \in 0,1 \forall k \\
C 4: \mathcal{F}_{k}^{\max }-\mathcal{F}_{k}^{\min } \leq F_{k}^{\lim }, \forall k
\end{array}
$$

We assume that for $\forall \epsilon, \Phi \in(\epsilon, 1], \exists \mathbb{P}^{\epsilon}=\{(\rho, \mathbf{p}) \mid$ $\Phi>\epsilon\}$, and the element number of $\mathbb{P}^{\epsilon}$ is denoted as $\left|\mathbb{P}^{\epsilon}\right|$.
It is trivial to verify that for $\forall \epsilon^{*} \geq \epsilon, \mathbb{P}^{\epsilon^{*}} \subseteq \mathbb{P}^{\epsilon}$, and $\left|\mathbb{P}^{\epsilon^{*}}\right| \leq$ $\left|\mathbb{P}^{\epsilon}\right|$. Thus, $\lim _{\epsilon \rightarrow 1}\left|\mathbb{P}^{\epsilon}\right|=\left|\mathbb{P}^{1}\right|=\min \left(\left|\mathbb{P}^{\epsilon}\right| \mid 0 \leq \epsilon \leq 1\right)$. It means $\mathbb{P}^{1}$ is the asymptotical minimal cover for problem (9). If $\mathbb{P}^{1}=\emptyset$, there is no optimal solution for problem (1); if $\left|\mathbb{P}^{1}\right|=1$, then $(\rho, \mathbf{p})$ is the optima we search for; if $\left|\mathbb{P}^{1}\right|>1,(\rho, \mathbf{p}) \in \mathbb{P}^{1}$ is Pareto optima for problem (9) and the global optima must be included in $\mathbb{P}^{1}$. Therefore, we can get an approximately equivalent optimization problem as follows:

$$
\left(\rho^{\dagger}, \mathbf{p}^{\dagger}\right)=\arg \max \Phi
$$

\section{Max-Min-SU}

Due to the noncontinuous of feasible set, generally can only find a set $\mathbb{P}^{\epsilon}$, such that $\Phi \in(\epsilon, 1]$. It is nontrivial to find out the Pareto-optimal front of set $\mathbb{P}^{\epsilon}$. The question becomes if there exist a equivalent function $\mathcal{F}$ such that $\left(\rho^{*}, p^{*}\right)=\arg \max \mathcal{F}$ if and only if $\left(\rho^{*}, p^{*}\right)=$ $\arg \left(\max \sum_{k=1}^{K} R_{k} \& \max \Phi\right)$. Max-Min-User is one of them.

Proposition 1. With the constraint of $\Phi=1$, if $\left(\rho^{*}, p^{*}\right)=$ $\arg \left(\max \sum_{k=1}^{K} R_{k}\right)$, then $\left(\rho^{*}, p^{*}\right)=\arg \max \min R_{k}$.

Proof. If $\exists\left(\rho^{\prime}, p^{\prime}\right)$, such that min $R_{k}^{\prime}<\min R_{k}$. According to (5) and $C 5$ in (9), $\sum_{k=1}^{K} R_{k}^{\prime}<\sum_{k=1}^{K} R_{k}$, which is contradictory with $\left(\rho^{*}, p^{*}\right)=\arg \left(\max \sum_{k=1}^{K} R_{k}\right)$, thus $\left(\rho^{\prime}, p^{\prime}\right)$ is not existed. Therefore, we can get an optimization problem equivalent to problem (8) as follows:

$$
\left(\rho^{*}, \mathbf{p}^{*}\right)=\arg \max \left(\min \left(R_{k}\right)\right)
$$

where the constraints are the same as in (10).

\section{Hybrid objective function}

As discuss above, we proposed two alternative objective functions MPF and Max-Min-SU. For MPF, we adopt method narrowing the feasible set to search for the approximate solution. This model has fast velocity of convergence since its aim is Pareto optima for problem (9), at the expense of quality of solution; for Max-Min-SU in a contrary, we derive that its solution is equivalent to the optimization problem (9). However, due to the uniqueness, the searching process is more different than that in MPF. Therefore, we propose a hybrid objective function that possesses advantages of both MPF and Max-MinSU. The format expression is as follows:

$$
\left(\rho^{*}, \mathbf{p}^{*}\right)=\arg \max \left(\min R_{k} \Phi\right)
$$

where the constraints are the same as in (10). And we will discuss the performance for the three fitness functions in Section "Particle swarm optimization based framework". 


\section{Problem decomposition}

Ideally, subchannel and power should be allocated jointly to achieve the optimal solution in (12). However, due to the mixed binary integer programming, it is prohibitive computational burden at CBS, which leads the increase of computation cost and allocation delay. Hence, we separates the problem (10) into subchannel allocation and power allocation to reduce the complexity, because the continuous variable $p_{k, n}^{i}$ and binary variable $\rho_{k, n}^{i}$ can be handled independently.

We first formulate subchannel allocation as an optimization problem with the assumption that all subchannels are allocated equal power distribution in each PRN, i.e.,

$$
p_{\sim, n}^{i}=\frac{1}{N_{i}} P_{\text {total }}^{i} \forall i
$$

where $p_{\sim, n}^{i}$ is the allocated power on subchannel $n$ of PRN $i$.

Thus the problem of subchannel allocation can be formulated as follows:

$$
\begin{gathered}
\rho^{*}=\arg \max \left(\min R_{k} \Phi\right) \\
\text { subject to : } C 1: \sum_{k=1}^{K} \rho_{k, n}^{i}=1, \rho_{k, n}^{i} \in\{0,1\}, \forall i \\
C 2: \mathcal{F}_{k}^{\max }-\mathcal{F}_{k}^{\min } \leq F_{k}^{\lim }, \forall k
\end{gathered}
$$

For a determined subchannel allocation, the optimization problem is formulated as follows:

$$
\mathbf{p}^{*}=\arg \max \left(\min R_{k} \Phi\right)
$$

subject to : $C 1: p_{k, m}^{i} \geq 0, \forall k, m, i$

$$
C 2: \sum_{k=1}^{K} \sum_{m=1}^{N_{i}} p_{k, n}^{i} \leq P_{\text {total }}^{i} \forall i
$$

\section{Penalty function}

The penalty function technique is an effective method to solve the constrained optimization problems [29]. By penalizing the constraints and building a single objective function, the constrained problem can be transformed into an unconstrained one, which is in turn maximized using an unconstrained optimization algorithm. A penalty function is generally defined as [30]:

$$
F(x)=f(x)+h(k) H(x), \quad x \in \mathbb{S}
$$

where $f(x)$ is the original objective function of the optimization problem. $h(k)$ is a dynamically modified penalty value, $k$ is the algorithm's current iteration number; $\mathbb{S}$ is the feasible set and $H(x)$ is a penalty factor, which is always problem dependent.
The definition of penalty function in (17) is additive. In this article, two penalty functions based on (17) to solve the constraint $\mathrm{C} 2$ in problem (15) and (16) can be formulated as follows.

\section{Additive penalty function}

$$
\Psi_{\text {add }}^{\text {channel }}=R_{k} \Phi-\lambda_{1} I_{\mathbb{R}^{+}}\left(\max _{1 \leq k \leq K}\left(F_{k}^{\mathrm{lim}}-\left(\mathcal{F}_{k}^{\max }-\mathcal{F}_{k}^{\text {min }}\right)\right)\right)
$$

$$
\Psi_{\text {add }}^{\text {power }}=R_{k} \Phi-\lambda_{2} I_{\mathbb{R}^{+}}\left(\max _{1 \leq i \leq S}\left(P_{\text {total }}^{i}-\sum_{m=1}^{N_{i}} \sum_{k=1}^{K} p_{k, m}^{i}\right)\right)
$$

where $I_{A}(x)$ is indicative function. $I_{A}(x)=0$ if $x \notin A$ and $I_{A}(x)=1$ if $x \in A . \lambda_{1}$ and $\lambda_{2}$ are the Lagrangian multipliers. In our problem, they are dependant on channel gains $h_{k, n}^{i}$ and power allocation results $p_{k, n}^{i}$, resulting in changing in every resource allocation. This will introduce great burden in CBS. To simplify this problem, we propose multiplicative penalty functions, which is independent with $h_{k, n}^{i}$ and $p_{k, n}^{i}$. Formally, the expressions of our proposed are written as follows.

\section{Multiplicative penalty function}

$$
\Psi_{\text {multi }}^{\text {channel }}=R_{k} \Phi I_{\mathbb{R}^{+}}\left(\max _{1 \leq k \leq K}\left(F_{k}^{\mathrm{lim}}-\left(\mathcal{F}_{k}^{\max }-\mathcal{F}_{k}^{\min }\right)\right)\right) \times \beta_{1}
$$

$$
\Psi_{\text {multi }}^{\text {power }}=R_{k} \Phi I_{\mathbb{R}^{+}}\left(\max _{1 \leq i \leq S}\left(P_{\text {total }}^{i}-\sum_{m=1}^{N_{i}} \sum_{k=1}^{K} p_{k, m}^{i}\right)\right) \times \beta_{2}
$$

where $\beta_{1}$ and $\beta_{2}$ are normalized penalty factors and enable penalty value to be adjusted dynamically. The expressions can be written as

$$
\begin{gathered}
\beta_{1}=\frac{2}{1+\exp \left(\frac{\max _{1 \leq k \leq K}\left|\left(\mathcal{F}_{k}^{\max }-\mathcal{F}_{k}^{\min }\right)-F_{k}^{\lim }\right|}{f_{N}-F_{k}^{\lim }}\right)} \\
\text { if }\left(P_{\text {total }}^{i}-\sum_{n=1}^{N_{i}} \sum_{k=1}^{K} p_{k, n}^{i}\right)>0, \forall i \\
\beta_{2}=1-\sqrt{\sum_{i=1}^{S}\left(P_{\text {total }}^{i}-\sum_{m=1}^{N_{i}} \sum_{k=1}^{K} p_{k, m}^{i}\right)^{2}}
\end{gathered}
$$


else

$$
\beta_{2}=0.9\left(1-\sqrt{\sum_{i=1}^{S}\left(P_{\text {total }}^{i}-\sum_{m=1}^{N_{i}} \sum_{k=1}^{K} p_{k, m}^{i}\right)^{2}}\right)
$$

By analysis above, we transform the problem (9) into an alternative with objective functions (20) (21) and constraint $C 1$ in (15) (16). In the next section, we will propose a heuristic algorithm to solve the alterative problem.

\section{Particle swarm optimization based framework}

The PSO algorithm was introduced by James Kennedy and Eberhart (1995) as an effective batch of heuristic algorithms [31]. Compared with other algorithms, PSO has better global searching ability at the beginning of the run and a local searching ability near the end of the run [32]. It is also effective for integer programming [33].

The standard PSO algorithm basically involves the following steps [31]:

(1) Construct the particle to map the solution of interest problem;

(2) Create the initial topology for swarm and parameters to initial the optimization;

(3) Calculate fitness value for each particle;

(4) Renew particle position;

(5) Return to (2) until the solution satisfies the requirement of interest problem.

Following the work, in this section we design a PSObased framework to solve the subchannel allocation and power allocation for $\mathrm{CR}$ system. At the beginning of Section "PSO structure", we discuss the design of particle structure to establish the mapping between PSO and resource allocation problem. Then, in order to improve the velocity of convergence, we propose an initial topology of swarm exploiting the channel characteristics in Section "Suboptimal power distribution for a fixed sub-channel allocation"; Section "Particle renew" discusses the particle renew and the process of the proposed algorithm.

\section{PSO structure}

\section{Suboptimal subchannel allocation}

Due to the constraint of proportional fairness, each SU should be allocated at least one subchannel in an resource allocation duration. Thus, we can exclude all of particles who do not traverse the set of SU. Furthermore, the subchannels allocated to one SU should be approximately bunching since the sampling ability is limited. So we can arrange SU sequentially and user $k$ is preallocated $\left|\Omega_{k}\right|$ subchannels as shown in Figure 3.

We name this particle as "base particle" because it is the start of particle structure for subchannel allocation and denote it as a $1 \times N_{\text {sensed }}$ dimension vector particle base $_{\text {. }}$ Later we will explain the relationship between "base particle" and "particle" who, in fact, executes the solution searching work.

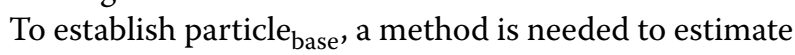
variable $\left|\Omega_{k}\right|$ for each SU. The capacity equation for SU $k$ on subchannel $n$ can be rewritten as follows:

$$
R_{k, n}=\log _{2}\left(1+p_{k, n}^{i} h_{k, n}^{2} / N_{0} \Gamma W\right)
$$

For the purposes of analysis, we define the virtual power $P$ as:

$$
P=p_{\sim, n}^{1}=\frac{P_{\text {total }}^{1}}{N_{1}}
$$

Thus we can get the expression as:

$$
p_{\sim, n}^{i}=\frac{P_{\text {total }}^{i} N_{1}}{P_{\text {total }}^{1} N_{i}} P, \quad \forall i
$$

We define

$$
H_{k, n}=\frac{h_{k, n}^{2}}{N_{0} \Gamma b} \frac{P_{\text {total }}^{i} N_{1}}{P_{\text {total }}^{1} N_{i}}
$$

as the virtual channel-to-noise ratio for user $k$ in subchannel $n$. Then (22) can be rewritten as:

$$
R_{k, n}=\log _{2}\left(1+P H_{k, n}\right)
$$

According to channel fading theory, large-scale fading is the main factor that affects the value of $H_{k, n}$. Hence, for the same SU, $H_{k, n}$ for all subchannel is approximate or fluctuate in the same level. Therefore, we can approximate function $\log (1+x)$ as linear if the variation of $x$ is small, as shown in Figure 4.

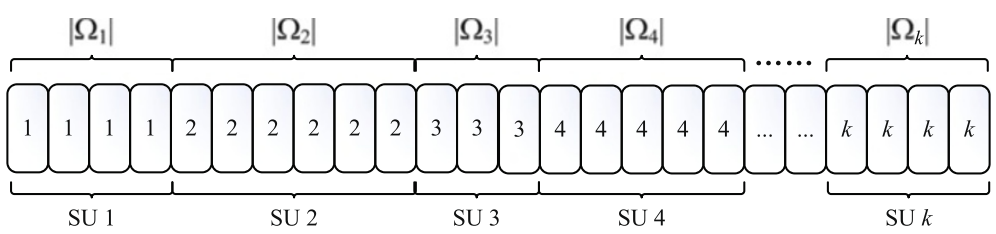

Figure 3 The base particle for subchannel allocation. 


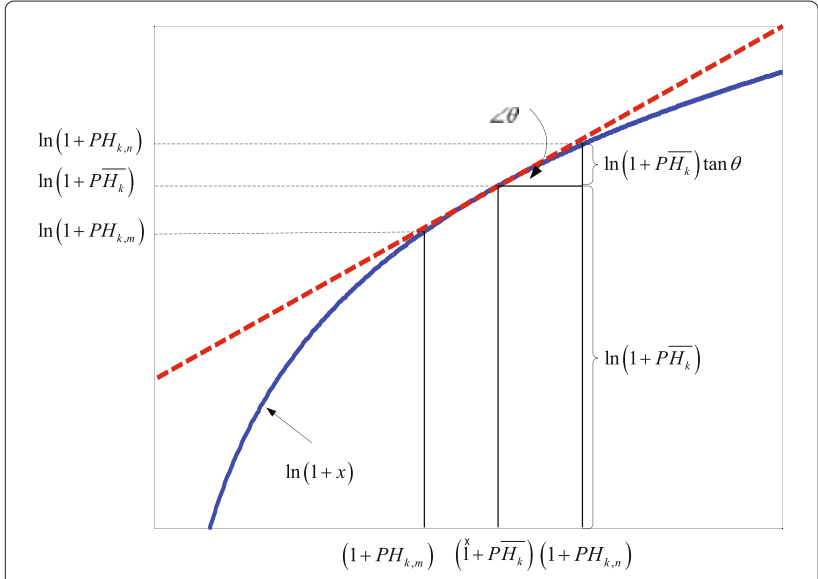

Figure 4 Illustration of approximation for Equations (24) and (25).

On the basis of the approximation, we can estimate the capacity sum for SU $k$ as:

$$
\begin{aligned}
& \frac{1}{\left|\Omega_{k}\right|} \sum_{n \in \Omega_{k}} \log _{2}\left(1+P H_{k, n}\right)=\frac{1}{\left|\Omega_{k}\right| \ln 2} \sum_{n \in \Omega_{k}} \ln \left(1+P H_{k, n}\right) \\
\approx & \frac{1}{\left|\Omega_{k}\right| \ln 2} \sum_{n \in \Omega_{k}}\left(P\left(H_{k, n}-\overline{H_{k}}\right) \tan \theta+\ln \left(1+P \overline{H_{k}}\right)\right) \\
= & \frac{1}{\left|\Omega_{k}\right| \ln 2}\left(\left|\Omega_{k}\right| \ln \left(1+P \overline{H_{k}}\right)+\sum_{n \in \Omega_{k}}\left(H_{k, n}-\overline{H_{k}}\right) \tan \theta\right) \\
= & \log _{2}\left(1+P \overline{H_{k}}\right)
\end{aligned}
$$

where $\overline{H_{k}}=\sum\left(H_{k, n}\right) / N^{\text {sense }}$ and

$$
\tan \theta=\frac{d\left(\ln \left(1+P \overline{H_{k}}\right)\right)}{d\left(1+P \overline{H_{k}}\right)}=\frac{1}{\left(1+P \overline{H_{k}}\right)}
$$

To satisfy the requirement of proportional fairness, we should make

$$
\left|\Omega_{1}\right| \frac{\log _{2}\left(1+P \overline{H_{1}}\right)}{\gamma_{1}}=\left|\Omega_{k}\right| \frac{\log _{k}\left(1+P \overline{H_{k}}\right)}{\gamma_{k}}, \quad \forall k
$$

Therefore, we can get the relationships as follows

$$
\begin{array}{r}
\left|\Omega_{2}\right|=\left|\Omega_{1}\right| \frac{\log _{2}\left(1+P \overline{H_{1}}\right) \gamma_{2}}{\log _{2}\left(1+P \overline{H_{2}}\right) \gamma_{1}} \\
\left|\Omega_{3}\right|=\left|\Omega_{1}\right| \frac{\log _{2}\left(1+P \overline{H_{1}}\right) \gamma_{3}}{\log _{2}\left(1+P \overline{H_{3}}\right) \gamma_{1}} \\
\ldots \\
\left|\Omega_{K}\right|=\left|\Omega_{1}\right| \frac{\log _{2}\left(1+P \overline{H_{1}}\right) \gamma_{k}}{\log _{2}\left(1+P \overline{H_{K}}\right) \gamma_{1}}
\end{array}
$$

and the total sensed subchannels $N^{\text {sense }}$ can be expressed as

$$
\begin{aligned}
N^{\text {sense }} & =\left|\Omega_{1}\right|+\left|\Omega_{2}\right|+\cdots+\left|\Omega_{K}\right| \\
& =\left|\Omega_{1}\right| \frac{\log _{2}\left(1+P \overline{H_{1}}\right)}{\gamma_{1}}\left(\sum_{k=1}^{K} \frac{\gamma_{k}}{\log _{2}\left(1+P \overline{H_{k}}\right)}\right)
\end{aligned}
$$

Substituting (23) into (24) and we can get the approximate $\left|\Omega_{k}\right|$ for each SU as:

$$
\left|\Omega_{k}\right|=\frac{N^{\text {sense }} \gamma_{k}}{\log _{2}\left(1+P \overline{H_{k}}\right)\left(\sum_{k=1}^{K} \frac{\gamma_{k}}{\log _{2}\left(1+P \overline{H_{k}}\right)}\right)}, \quad \forall k
$$

particle $_{\text {base }}$ is not optimal because we just arrange SU order in a sequential way. Intuitively, we can use sorting algorithm to rearrange SU order to achieve higher objective (fitness) value. Bubble Sorting is a classical sorting algorithm with complexity $O\left(n^{2}\right)$. In this article, we use a modified bubble sorting algorithm to rearrange SU order. We denote the SU order as a $1 \times K$ dimension vector $\mathbf{K}$ and the element of $\mathbf{K}$ indicates the $S U$ index. fitness $_{\mathbf{K}}$ represents the fitness value with the SU order K and can be calculated by (20). The process is depicted in the following:

\section{Algorithm $1 \mathrm{SU}$ sorting algorithm}

Generate $\mathbf{K}$ in a random permutation;

for $k=1$ to $K-1$

for $l=1$ to $K-k-1$

Calculate fitness $\mathbf{K}$ by (20);

$\mathbf{K}_{\text {temp }} \leftarrow \mathbf{K}$

$\mathbf{K}_{\text {temp }}(l) \leftarrow \mathbf{K}(l+1)$

$\mathbf{K}_{\text {temp }}(l+1) \leftarrow \mathbf{K}(l)$

Calculate fitness $\mathbf{K}_{\mathrm{temp}}$ by (20);

if fitness $\mathbf{K}_{\text {temp }}>$ fitness $_{\mathbf{K}}$

$$
\mathbf{K} \leftarrow \mathbf{K}_{\text {temp }}
$$

else

continue;

end for

end for

We denote the result of sorting as particle ${ }_{\text {base }}^{\text {opt }}$. The feasible set in this initial topology is only $K$ !, which is much less than the whole feasible set $K^{N-m} C_{K-1}^{N-m}$. The

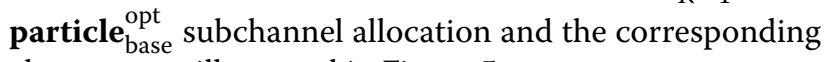
elements are illustrated in Figure 5.

It is an intuitive idea to generate particle based on particle $_{\text {base }}^{\text {opt }}$. However, since variable $K$ is usually much smaller than $N^{\text {sense }}$, the activity of particle with variable SU index is limited extremely. Furthermore, with the renew of such particle, it is difficult to control the solution 


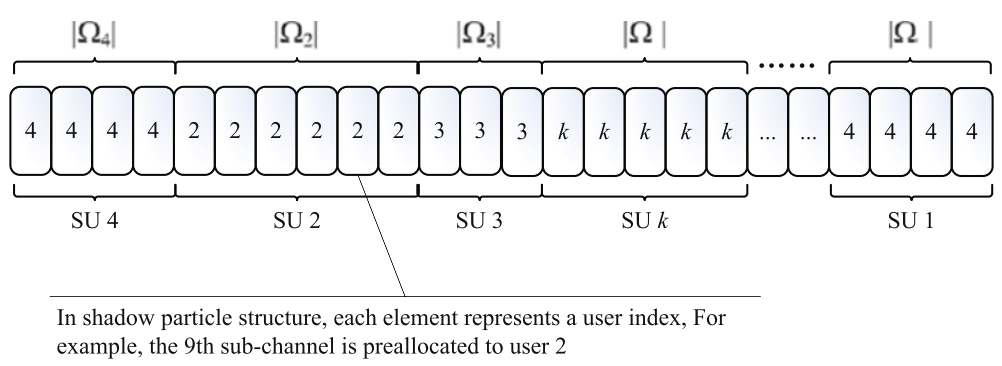

Figure 5 The optimized base particle for subchannel allocation.

under the constraint $C 4$. Therefore, we design a particle with variable space $\left[0, N^{\text {sense }}\right]$. First, we generate a $1 \times N^{\text {sense }}$ dimension particle, and denoted as particle $_{\text {init }}$ :

$$
\operatorname{particle}_{\text {init }}(n)=|\delta-n|, \quad \forall n
$$

where $\delta \in\left\{\delta:\right.$ particle $_{\text {base }}(\delta)=$ particle $\left._{\text {base }}^{\text {opt }}(n), 1<\delta<K\right\}$.

Based on particle init $_{\text {, we can generate a set of } 1 \times N^{\text {sense }}}$ dimension vector particle as follows:

$$
\begin{aligned}
& \text { - } \text { for } i=1 \text { to } N^{\text {pso }} \\
& \text { - } \quad \text { for } n=1 \text { to } N^{\text {sense }}
\end{aligned}
$$

$$
-\operatorname{particle}_{i}(n)=\text { particle }_{\text {init }}(n)+\omega
$$

- end for

- end for

where $N^{\text {pso }}$ is the number of particles, particle ${ }_{i}$ refer to the $i$ th particle. $\omega$ follows uniform distribution in $\left[0, N^{\text {sense }}\right]$.

It is noticed that the elements of particle are no physical significance. To calculate the fitness value for each particle, we should transform the vector particle into a vector with the subchannel allocation information. Therefore, we define a vector, namely particle shadow $_{\text {corre- }}$ sponding to particle. The mapping relationship between particle $_{\text {shadow }}$ and particle can be written as:

$$
\begin{gathered}
\delta=\bmod \left(n+\operatorname{particle}_{i}(n), N^{\text {sense }}\right) \\
\text { particle }_{\text {shadow }, i}(n)=\text { particle }_{\text {init }}(\delta)
\end{gathered}
$$

where $\bmod (x, y)$ represents the operation of modulus after division and we define special point $\bmod (x, y)=x$ if $x / y=m, m$ is an arbitrary integer. particle shadow,$i(n)$ indicates the $n$th element of the $i$ th particle shadow $_{\text {. }}$

The reason why we call the particle "shadow particle" lies in that each shadow particle maps a particle who is responsible for searching optimum. To illustrate the relationships among these particles, Figure 6 shows the transformations by taking an example with 8 SUs and 8 subchannels case.

The $R_{k}$ in the particle ${ }_{i}$ can be obtained as:

$$
R_{k}=\sum_{\text {particle }_{\text {shadow }, i}(n)=k} \log \left(1+P H_{k, n}\right), \quad \forall k
$$

Substituting (32) into (20) and we can get the fitness $(i)$ of the $i$ th particle.

\section{Suboptimal power distribution for a fixed sub-channel allocation}

Once suboptimal subchannel allocation is determined, we should restructure the particle, since the requirement has been changed in power allocation. Each element in vector particle $_{\text {shadow }}(n)$ is changed to represent a power value. For example, the fourth subchannel is allocated to power $0.011 \mathrm{~W}$ and the transmission rate for each SU can be calculated by joint subchannel and power allocation results, as shown in Figure 7.

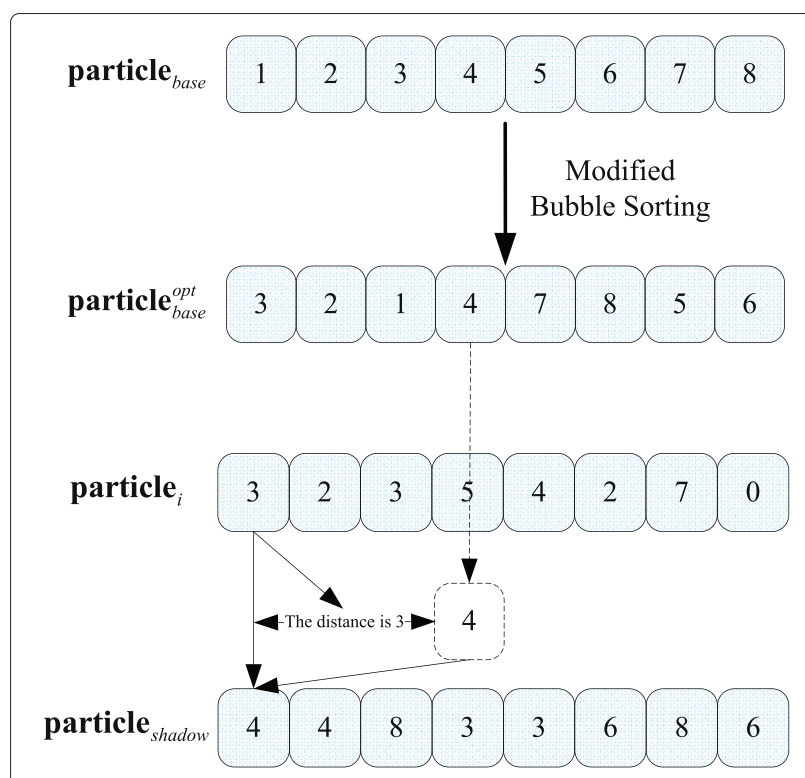

Figure 6 Illustration for particle transformations. 


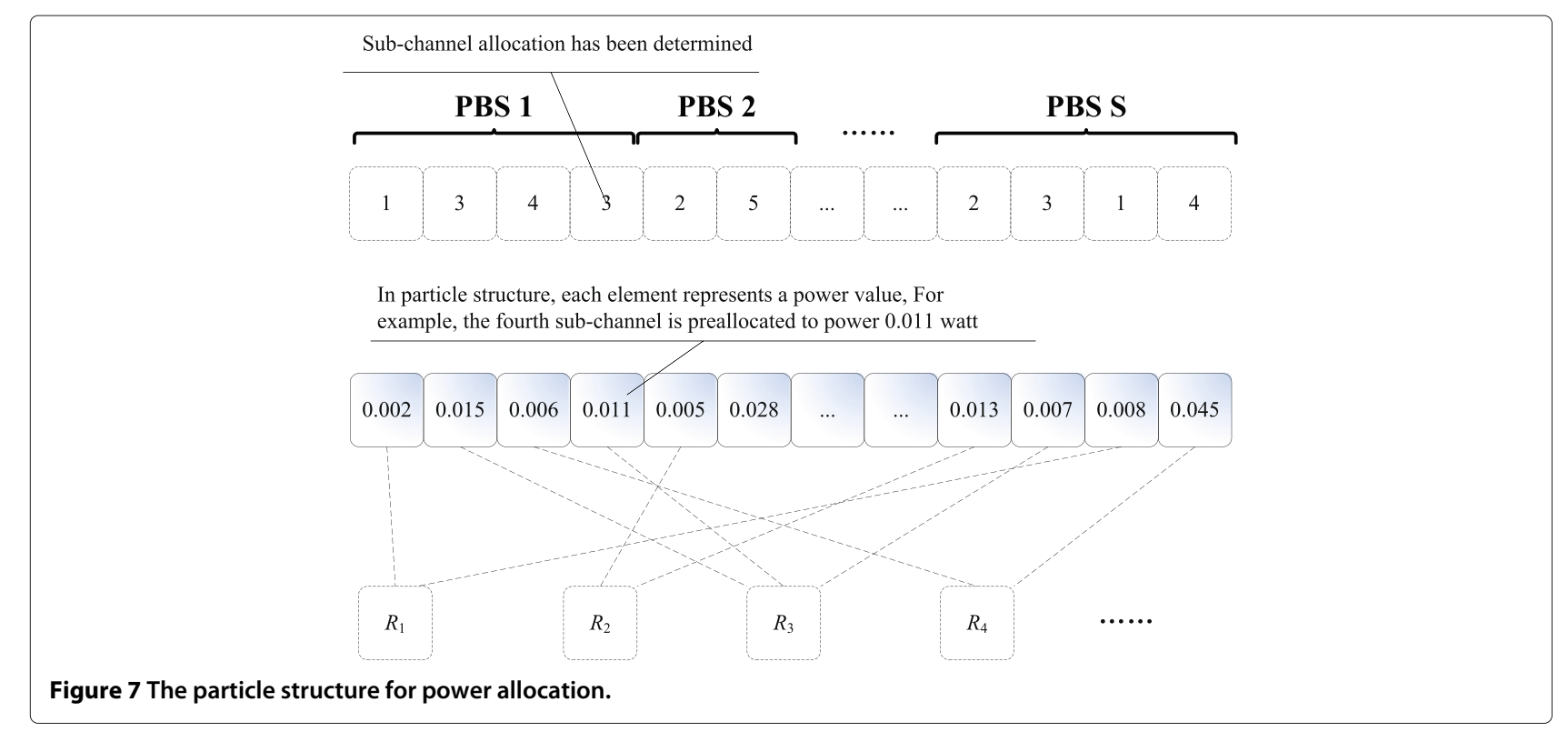

\section{Particle renew}

According to the objective function, the search space we focus is $N^{\text {sense }}$-dimensional, the $i$ th particle of the swarm is represented by a $N^{\text {sense }}$-dimensional vector particle . $_{i}$. The best particle of the swarm, i.e., the particle with the highest fitness value, is denoted by Gparticle, and its fitness value is denoted as $G_{\text {best }}$. The best previous fitness value and corresponding position of the particle pis $_{i}$ recorded and represented as $P_{\text {best }}^{i}$ and Pparticle P $_{i}$ and the position change of the $i$ th particle is denoted as velocity . The particles are manipulated according to the following equations:

$$
\begin{array}{r}
\text { velocity }_{i}=\chi\left(\text { velocity }_{i}\right. \\
+c_{1} \omega_{1}\left(\text { Pparticle }_{i}-\text { particle }_{i}\right) \\
\left.+c_{2} \omega_{2}\left(\text { Gparticle }_{-} \text {particle }_{i}\right)\right)
\end{array}
$$

$$
\text { particle }_{i+1}=\text { particle }_{i}+\text { velocity }_{i}
$$

where $\chi$ is inertia coefficient in PSO algorithm and the variables $\omega_{1}$ and $\omega_{2}$ are random positive numbers, drawn from a uniform distribution within interval $[0,1]$. An extensively employed function of $\chi, c_{1}$, and $c_{2}$ is presented in [34]:

$$
\chi=\frac{2}{\left|2-c-\sqrt{c^{2}-4 c}\right|}, \quad c=\left(c_{1}+c_{2}\right)>4
$$

where $c=4.1, \chi c_{1}=\chi c_{2}=1.149445$, and so $\chi \approx 0.729$ are recommended.
Therefore, the PSO-based resource allocation can be described as follows:

\section{Algorithm 2 PSO-based adaptive resource allocation algorithm}

Step 1: Initialization

for $i=1$ to $S$

sensingSpectrum $(i)$

end for

calculate $\left|\Omega_{k}\right|$ for all $k$ according to (28);

generate base particle according to Algorithm 1

generate particle and shadow particle according to (30) (31)

Step 2: Subchannel Allocation

for $i=1$ to $N^{\text {pso }}$

calculate the fitness $(i)$ according to (32)

end for

renew $G_{\text {best }}$, Gparticle, $P_{\text {best }}^{i}$ and Pparticle Pp $_{i} \forall i$

renew velocity and particle $_{i} \forall i$ according to (33) (34)

if satisfy the stop conditions

go to Step 3

else

go to the top of Step 2;

end if

Step 3: Power Allocation

Transform particle according to Section "Max-MinSU";

for $i=1$ to $N^{\text {pso }}$

calculate the fitness $(i)$ according to (32)

end for

renew $G_{\text {best }}$, Gparticle, $P_{\text {best }}^{i}$ and Pparticle Pp $_{i} \forall i$

renew velocity and particle $_{i} \forall i$ according to (33) (34)

if satisfy the stop conditions 


\author{
stop algorithm \\ else \\ go to the top of Step 3; \\ end if
}

\section{Computational complexity analysis}

According to the analysis above, the computational complexity of proposed algorithm can be segmented into four parts: initialization, initialization topology of swarm, subchannel allocation, and power allocation, where subchannel allocation and power allocation can be further segmented into adapting and updating parts.

For initial stage, the algorithm should calculate the achievable rate of each SU in every subchannel. This cost complexity of $O\left(K \times N^{\text {sense }}\right)$. After that, sorting algorithm expenses $O\left(K^{2}\right)$ complexity, and in each sorting, the algorithm also needs to calculate $O\left(K \times N^{\text {sense }}\right)$ computation unit. Hence, the total complexity in initial topology is $O\left(K^{2} \times K \times N^{\text {sense }}\right)$. In the stage of adapting of subchannel, the proposed algorithm needs to calculate fitness value of each particle, which cost totally $O\left(K \times N^{\text {sense }} \times N^{\text {pso }}\right)$ for all particles. Then, in order to renew the $G_{\text {best }}$ and $P_{\text {best }}$ for all particles, comparison operations with complexity $O\left(N^{\mathrm{pso}}\right)$ is needed. At the end of one searching in subchannel, a particle renewing with the complexity of $O\left(N^{\text {sense }} \times N^{\text {pso }}\right)$ is executed. The complexity analysis for power allocation is the same as subchannel allocation and the corresponding results for each step is listed in Table 1.

\section{Simulation results and discussion}

In this section, we present the simulation results to show the performances of the proposed resource allocation algorithm.

\section{Simulation environment}

To evaluate the performance of proposed algorithm in heterogeneous environment, we consider a system consisting of one CRN and three ambient PRNs as shown in Figure 8. A CBS is located in the center of the map with

Table 1 Computational complexity upper bounds of four algorithms

\begin{tabular}{ll}
\hline Segment & Computational complexity \\
\hline Initialization & $O\left(K \times N^{\text {sense }}\right)$ \\
Initialization topology & $O\left(K^{2} \times K \times N^{\text {sense }}\right)$ \\
Adapting of subchannel & $O\left(K \times N^{\text {sense }} \times N^{\text {pso }}\right)$ \\
Gbest and $P_{\text {best }}^{i}$ Renew of subchannel & $O\left(N^{\text {pso }}\right)$ \\
Updating of subchannel & $O\left(N^{\text {sense }} \times N^{\text {pso }}\right)$ \\
Adapting of power & $O\left(K \times N^{\text {sense }} \times N^{\text {pso }}\right)$ \\
Gbest and $P_{\text {best }}^{i}$ Renew of Power & $O\left(N^{\text {pso }}\right)$ \\
Updating of power & $O\left(N^{\text {sense }} \times N^{\text {pso }}\right)$ \\
Total & $O\left(K^{3} \times N^{\text {sense }} \times N^{\text {pso }}\right)$ \\
\hline
\end{tabular}

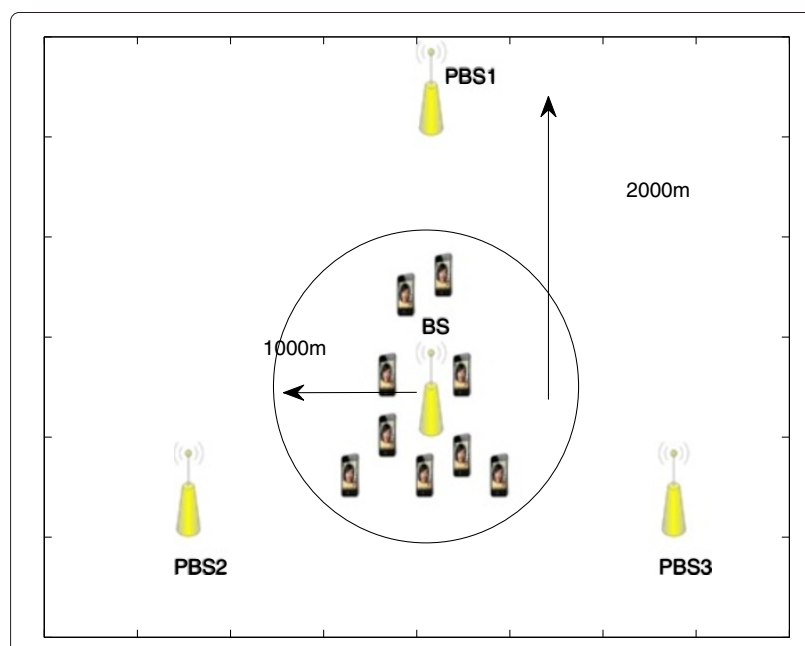

Figure 8 Simulation topology for the infrastructure-based CRN with multiple primary networks.

the coverage radius $d=1000 \mathrm{~m}$. Three PBSs are arranged around the CBS as vertex of isosceles triangle, like a typical cell's structure. The distance between CBS and some PBS is $2 d=2000 \mathrm{~m}$. We select the long-term evolution (LTE) as the background PRNs. According to the proposal of IEEE 1900, LTE network will adopt the DSA technique, and all radios are equipped with cognitive functionality in the near future $[35,36]$.

In all simulations presented in this section, the COST231 Hata path loss model is considered [37].

$$
\begin{aligned}
P L[d B]= & \left(44.9-6.55 \log \left(h_{\mathrm{BS}}\right)\right) \log (d / 1000)+46.3 \\
& +\left(35.46-1.1 h_{\mathrm{MS}}\right) \log \left(F_{i}\right)-13.82 \log \left(h_{\mathrm{BS}}\right) \\
& +0.7 h_{\mathrm{MS}}+C
\end{aligned}
$$

where $h_{\mathrm{BS}}$ and $h_{\mathrm{MS}}$ are the height of base station and mobile station, respectively. Without loss of generality, we assume $h_{\mathrm{BS}}=32 \mathrm{~m}$ and $h_{\mathrm{MS}}=1.5 \mathrm{~m} ; F_{i}$ is the central transmission frequency. In this article, three PRNs are assumed to be licensed in LTE potential band, with central frequency $F_{1}=1805 \mathrm{MHz}, F_{2}=1930 \mathrm{MHz}$ and $F_{3}=$ $2110 \mathrm{MHz}$, respectively. The shadowing is implemented by lognormal distribution with standard deviation values of $6 \mathrm{~dB}$. Each PRN share $20 \mathrm{MHz}$ spectrum with CRN in overlay mode. We assume that the subchannel spacing is approximate to $180 \mathrm{kHz}$, which is equal to one resource block (RB) in LTE network and hence the maximal available subchannels in each PRN is about $110(19.8 \mathrm{MHz})$. For small-scale fading, we adopt Clarke's flat fading model with six independent Rayleigh multipaths the same as in [14]. The power delay profile is assumed exponentially decaying with $e^{-2 l}$, where $l$ is the multipath index. Hence, 
the relative power of the six multipath components are $[0,-0.869,-17.37,-26.06,-34.74,-43.43]$.

We assume that the maximal passband for $\mathrm{SU}$ is $200 \mathrm{MHz}$, which usually needs approximate $1000 \mathrm{MSPS}$ or higher A/D converter in engineering implementation. The total transmission power of CRN on different PRNs is dependent on the results of negotiation between PRN and CRN. How to evaluate the utility of spectrum trading is out of our scope, but in order to reflect differences, we assume that the power limits in PRNs is different. Specifically, we adopt $P_{\text {total }}^{1}=5 \mathrm{~W}, P_{\text {total }}^{2}=10 \mathrm{~W}$ and $P_{\text {total }}^{3}=$ $5 \mathrm{~W}$, respectively. All other simulation parameters are list in Table 2.

\section{Results and discussion}

\section{Comparisons with different objective functions}

Figure 9 shows the performance comparisons of three objective functions discussed in Section "Objective function", with $K=20$ and $N^{\text {sense }}=200$. Figure 9a,b illustrates the convergence process in subchannel allocation and power allocation respectively, and Figure 9c indicates the final capacity allocation of each SU. In Figure 9, we can see that the MAX-MIN-SU scheme performs worst in terms of proportional fairness, minimum user's capacity and sum capacity. The reason lies in that the objective of the MAX-MIN-SU is unique, which results in that the search process is sensitive to the initial conditions and prone to be trapped in a local minimum. The

Table 2 Simulation parameter list

\begin{tabular}{ll}
\hline Parameter & Value \\
\hline Number of PRN: $S$ & 3 \\
Distance between CBS and PBS: $2 d$ & $2000 \mathrm{~m}$ \\
Height of base station $h_{\mathrm{BS}}:$ & $32 \mathrm{~m}$ \\
Height of mobility station: $h_{\mathrm{MS}}$ & $1.5 \mathrm{~m}$ \\
Number of SU: $K$ & $10-30$ \\
Total power limit of PRN 1: $P_{\text {total }}^{1}$ & $5 \mathrm{~W}$ \\
Total power limit of PRN 2: $P_{\text {total }}^{2}$ & $10 \mathrm{~W}$ \\
Total Power Limit of PRN 3:P $P_{\text {total }}^{3}$ & $5 \mathrm{~W}$ \\
Center frequency of PRN 1: $F_{1}$ & $1805 \mathrm{MHz}$ \\
Center frequency of PRN 2: $F_{2}$ & $1930 \mathrm{MHz}$ \\
Center frequency of PRN 3: $F_{3}$ & $2110 \mathrm{MHz}$ \\
Maximal available bandwidth: & $330 \mathrm{MHz}$ \\
Bandwidth of each subchannel: $W$ & $180 \mathrm{kHz}$ \\
Bandpass limit of SU: $F_{k}^{\text {lim }} \forall k$ & $200 \mathrm{MHz}$ \\
BER limit of SU: BER min & $10^{-3}$ \\
Proportional fairness: $\Phi$ & 0.99 \\
Number of particle: $N$ Pso & 200 \\
Maximal iteration of subchannel & 100 \\
Maximal iteration of power & 100 \\
\hline
\end{tabular}

MPF scheme has the best proportional fairness $(\Phi \geq 0.98)$ and the convergence process is smooth, both in subchannel allocation and power allocation. As we analyze in Section "Objective function ", the MPF objective is the Pareto optima for problem (9), hence the search process converges faster.

As shown in Figure 9, the proposed hybrid scheme outperforms the other two schemes in terms of both minimum user's capacity and sum capacity. This is because that the hybrid scheme combines the advantages of the MPF and the MAX-MIN-SU schemes. Specifically, the proposed scheme possesses a medium convergence ability with slight fluctuations (as shown in Figure 9a), which enables the search process jumping out the local traps with high probability; furthermore, with the consideration of $\max \min R_{k}$, the hybrid scheme can also keep the quality of solution in a high level. Figure 9c shows the final resource allocation results with three schemes. We can see that the hybrid scheme has a higher capacity than the other two schemes for most SUs. Therefore, the proposed hybrid objective function can provide better system performance and user experience.

In addition to the comparisons of different objective functions, two other results should be noticed as well. First, we can see that the MPF scheme outperforms the hybrid scheme in terms of proportional fairness while the hybrid scheme outperforms the MPF schemes in terms of capacity. This result reflects the tradeoff between the proportional fairness and the sum capacity, which is consistent with the conclusion in [14]. Second, as shown in Figure 9b, we can see that the improvement of system performance is limited by power allocation (less than 10\%). Therefore, we can remove the power allocation process with little performance loss, if the system is subject to the computational complexity constraint.

\section{Comparisons with the conventional algorithm}

To the best of the authors' knowledge, there is no research jointly considering such constraints simultaneously. One similar study was presented in [14]. The authors of [14] proposed an algorithm to solve the resource allocation problem considering proportional fairness. However, they ignored the bandpass limit of users in [14]. To ensure the algorithm in [14] suitable for broadband spectrum environment, we have to modify it with the constraint of bandpass limit of SU. We assume that each SU in the algorithm in [14] can operate simultaneously over two PRNs, in other words, the maximal frequency distance for each $\mathrm{SU}$ is $220 \mathrm{MHz}$ in our simulation scenario. Then, we divide all $K$ SUs into two parts, SUs from 1 to $K / 2$ are grouped as "first half" and SUs from $K / 2+1$ to $K$ are grouped as "second half". Each PRN includes SUs as shown in Figure 10. Based on the modification, we can 


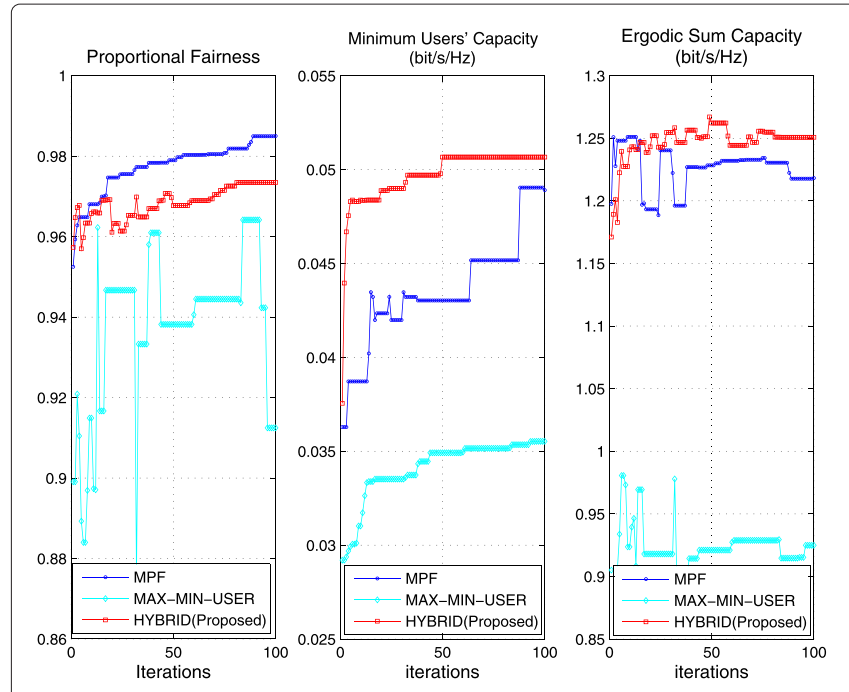

(a) Convergence in Subchannel Allocation
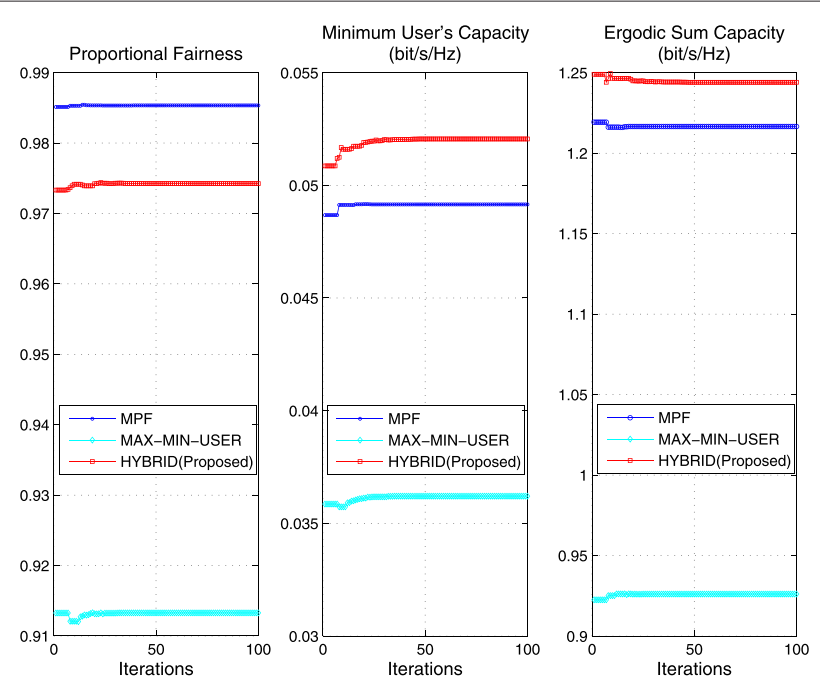

(b) Convergence in Power Allocation

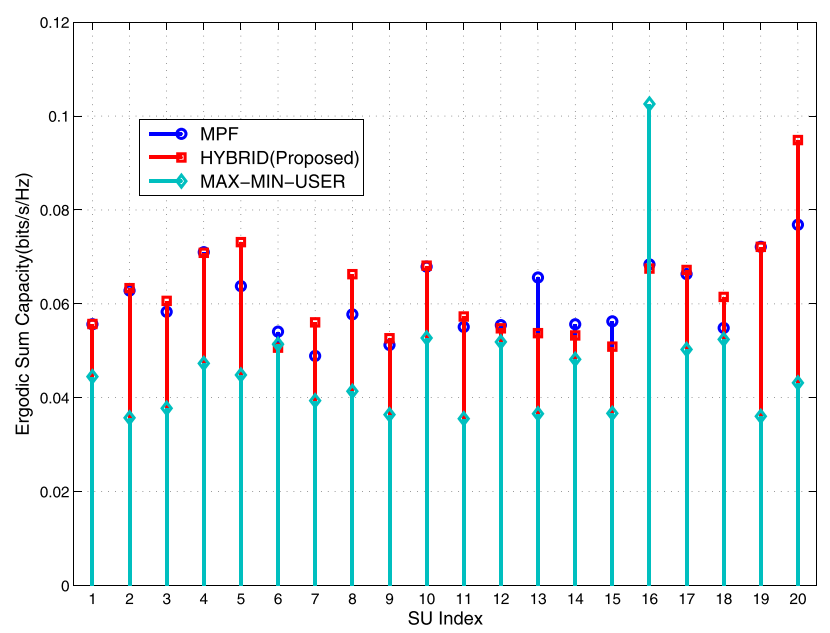

(c) Final Resource Allocation Results

Figure 9 Performance comparisons with three objective functions.

allocate the subchannels and power in each PRN using Zukang's algorithm independently.

In Figure 11, we show the minimum SU's capacity versus the number of $\mathrm{SU}$ with the proposed algorithm and the algorithm in [14]. The spectrum utilization refers to the spectrum occupancy percentage of primary systems. Specifically, the spectrum utilization $50 \%$ means the available idle subchannels is $(1-0.5) N \approx 165$ and the

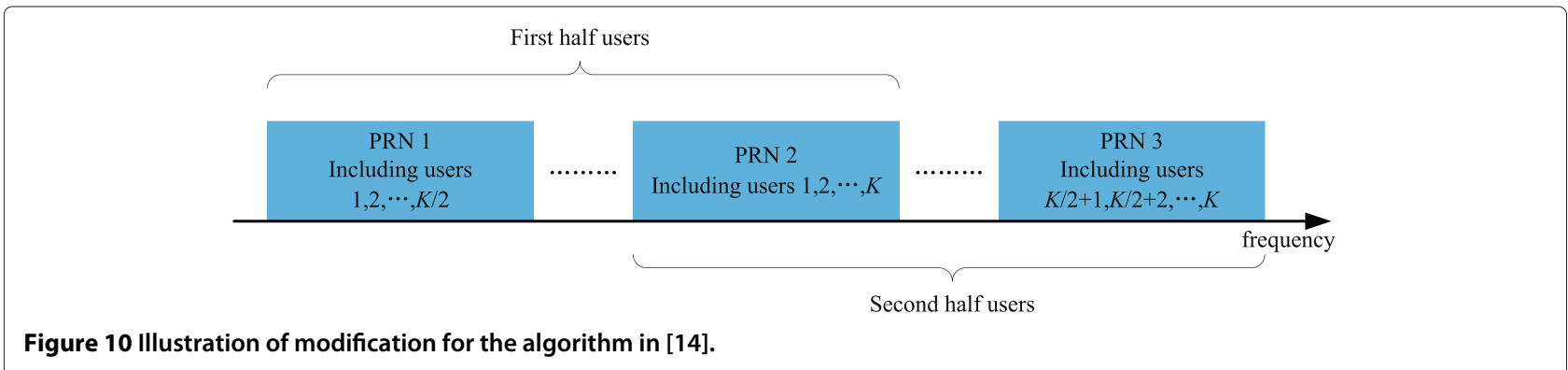




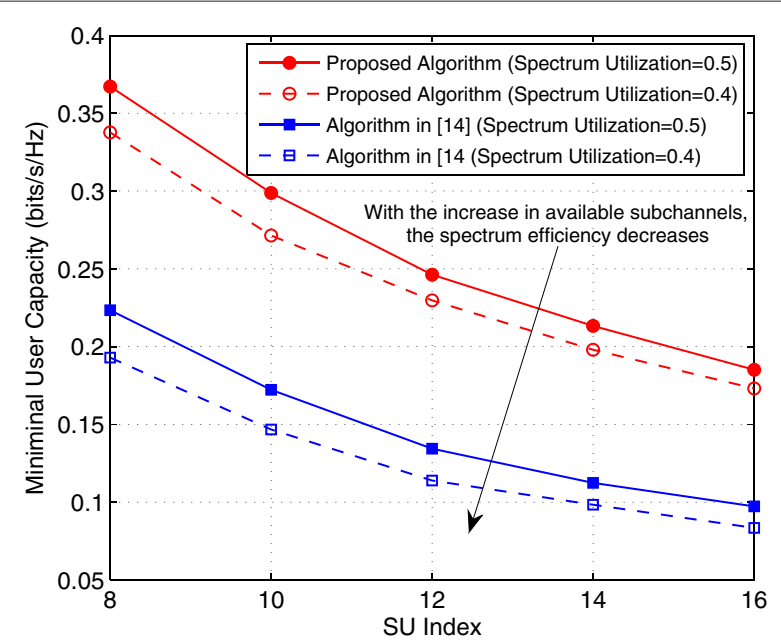

Figure 11 Minimum SU's capacity versus $K$ and spectrum utilization.

spectrum utilization $40 \%$ means the available idle subchannels is $(1-0.4) N \approx 198$. As shown in Figure 11 , the proposed algorithm achieves significant capacity gain over the algorithm in [14] under the constraint of bandpass limit. The reason is that in the proposed algorithm, SUs can be allocated to subchannels more flexibly than that in the algorithm in [14]. In addition, we can observe that the minimum capacity decreases with the number of available subchannels. This is because low power is allocated to each subchannel under a given maximum power as the number of subchannel increases.

However, as shown in Figure 12, we note that achievable transmission rate actually increases with the number of available subchannel for SUs. For example, when $K=8$,

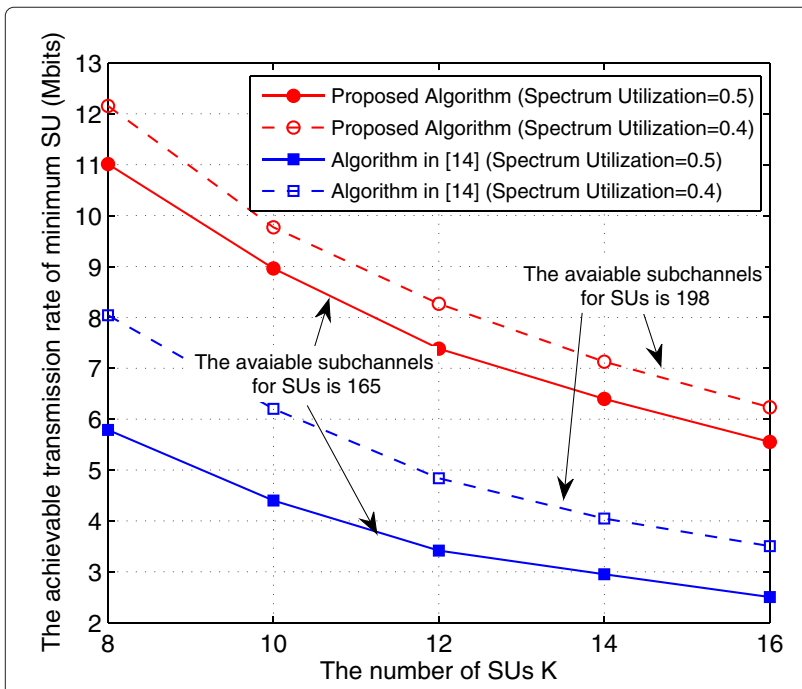

Figure 12 Minimum SU's achievable rate versus $K$ and spectrum utilization.

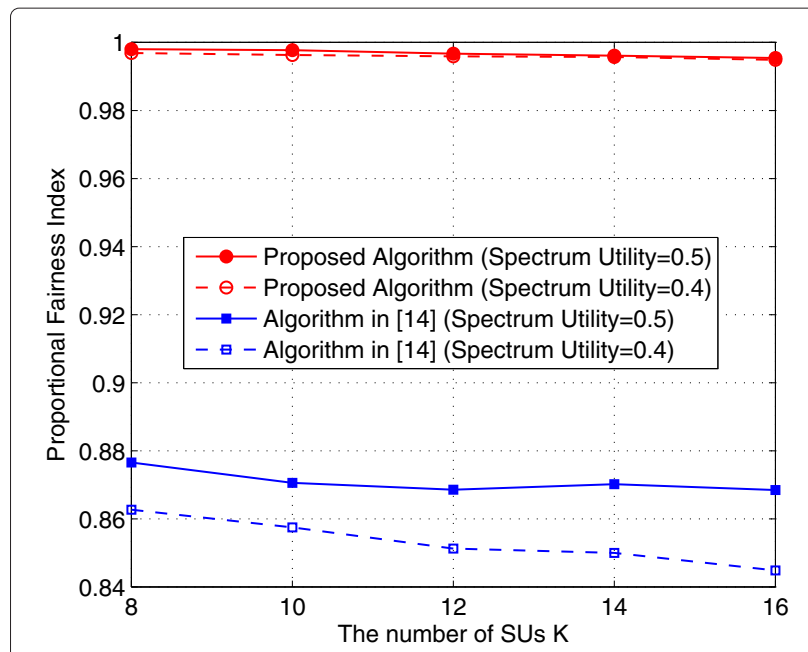

Figure 13 Proportional fairness versus $K$ and spectrum utility.

the total transmission rate with $N^{\text {sense }}=198$ (spectrum utilization is 0.4) is $12.16 \mathrm{Mbits}$, while the total rate is 11.01 Mbits with $N^{\text {sense }}=165$ (spectrum utilization is 0.5 ).

Figure 13 shows the comparisons of proportional fairness with the two algorithms. It can be seen that the proposed algorithm outperforms the algorithm in [14] in terms of proportional fairness, with $\Phi>0.98$ for all $K$. This is because the proposed algorithm can allocate subchannels and power to SUs in a global viewpoint, whereas Zukang's algorithm is constrained by the division of SU groups. Figure 14 shows the capacity distribution among SUs for one channel realization. It also shows the phenomenon that the capacity is unbalanced between the first-half group and the second-half group. In addition, we can see that with the increase in the number of SUs, the

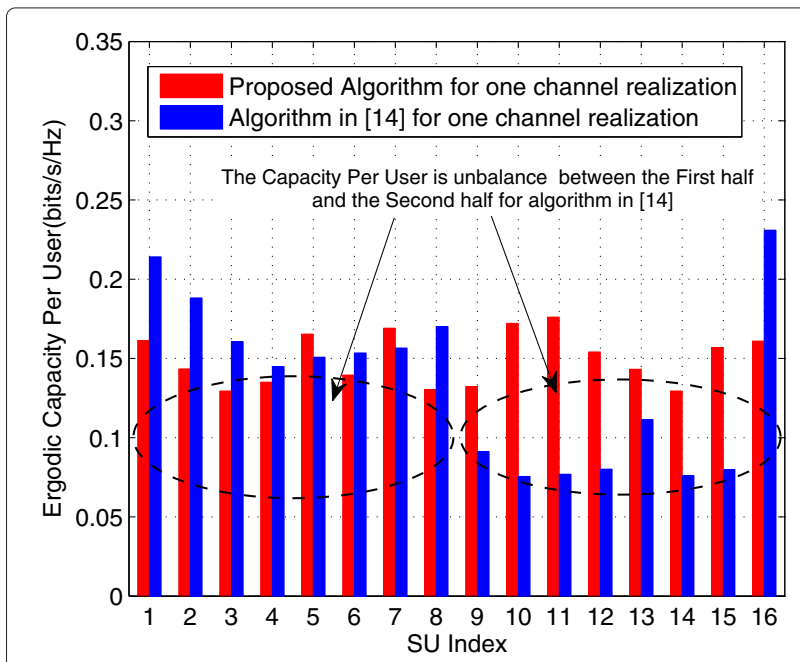

Figure 14 Capacity distributions for one channel realization. 
proportional fairness of CRN system decrease sightly. In the light of (5), the more the number of SUs is ongoing in the system, the harder the condition $\Phi=1$ is achieved.

\section{Impact by spectrum sensing errors}

The imperfect performance of a spectrum sensing algorithm is also a significant effect on the usage of the spectrum resources for CRNs. In this section, we take an energy detector for example to illustrate the impact of the spectrum sensing errors on the transmission rate for SUs. The $P_{\mathrm{f}}$ and $P_{\mathrm{d}}$ can be expressed as [38]

$$
\begin{aligned}
& P_{f}=Q\left(\frac{\gamma-\sigma^{2}}{\sqrt{\frac{2}{m}} \sigma^{2}}\right) \\
& P_{m}=1-Q\left(\frac{\gamma-\left(P+\sigma^{2}\right)}{\sqrt{\frac{2}{m}}\left(P+\sigma^{2}\right)}\right)
\end{aligned}
$$

where $m$ is the number of sampling points, $\gamma$ is the detector threshold and $Q(\cdot)$ is the standard Gaussian complementary CDF. $P$ is the received average signal power and $\sigma^{2}$ represents the noise variance, and thus the average SNR is $P / \sigma^{2}$.

Figure 15 shows the achievable transmission sum rate $\left(R \cdot N^{\text {sense }} \cdot W\right)$ versus $P_{f}$, where "dash line" represents the sum rate calculated by the ARA algorithm. In practice, the CBS cannot judge which subchannels have been occupied by PRNs because of sensing errors. Therefore, the sum rate is calculated without considering the interference from PRNs. "solid line" represents the allocation results with the interference from PRNs. As shown in Figure 15, the gap between the sum rate calculated by the CBS and the actual achievable sum rate decreases with $P_{\mathrm{f}}$. The reason is when $P_{\mathrm{f}}$ increases, we can achieve lower $P_{\mathrm{m}}$,

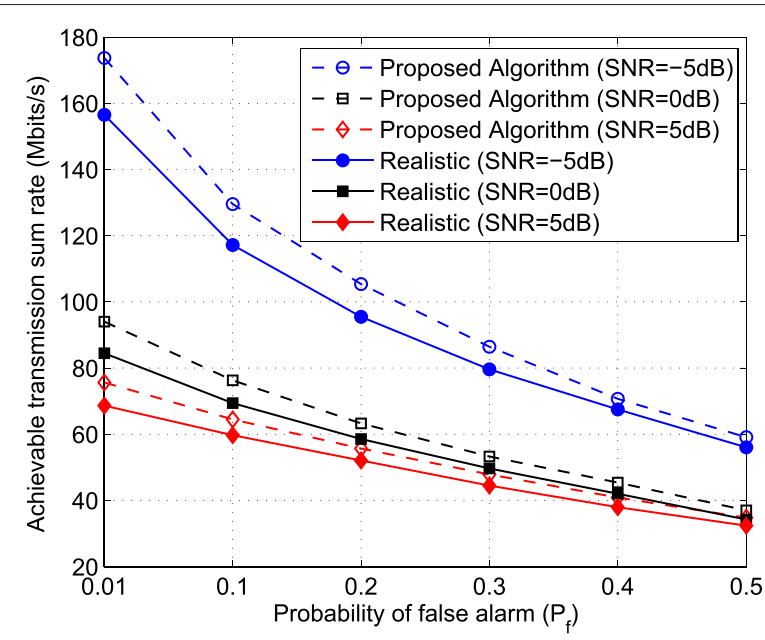

Figure 15 Achievable transmission sum rate versus SNR and $P_{\mathrm{f}}$.

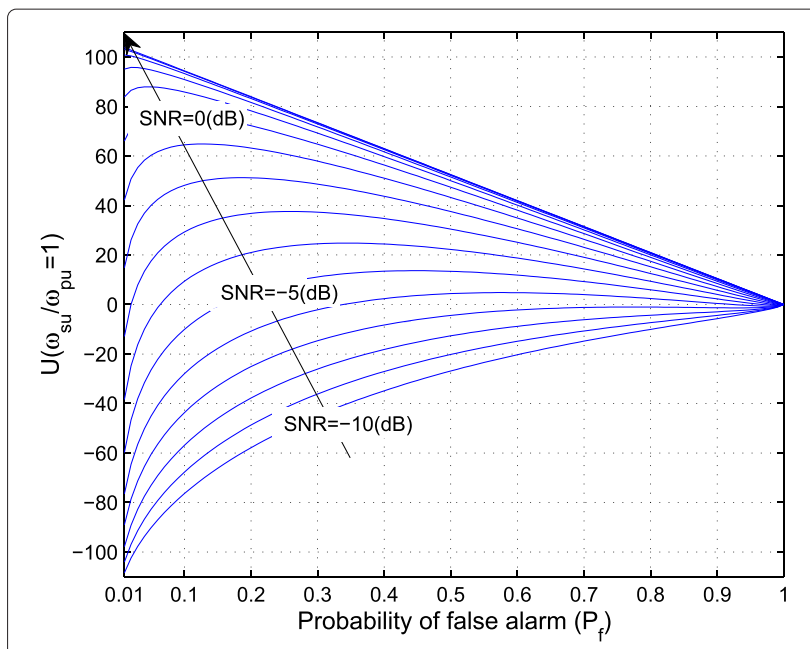

Figure 16 Utility function $U$ versus SNR and $P_{f}$.

resulting in the calculation error caused by PRNs interference reduces. This means that the ARA algorithm is more credible with lower $P_{\mathrm{m}}$.

In addition, for a given SNR, with the increase in $P_{\mathrm{f}}$, the number of $N^{\text {sense }}=\left(1-P_{f}\right) N^{\text {idle }}+P_{m} N^{\text {busy }}$ reduces. Hence, when $P_{\mathrm{f}}$ is small, the CRN gains higher achievable transmission sum rate. However, it is at the expense that more subchannels occupied by PRNs are miss detected. Therefore, there exists an optimal $P_{\mathrm{f}}$ to balance the tradeoff between the system performance of CRN and interference on PRNs. How to obtain the solution of the optimal $P_{\mathrm{f}}$ is out of our scope of this article, and we just define a simple utility function $U$ as an example to illustrate the existence of the tradeoff.

$$
U=\omega_{s u} \cdot\left(1-P_{f}\right) N^{\text {idle }}-\omega_{p u} \cdot P_{m} N^{\text {busy }}
$$

The utility function $U$ consists of two parts, where $\omega_{\text {su }} N^{\text {idle }}\left(1-P_{\mathrm{f}}\right)$ is reward part and $\omega_{\mathrm{pu}} \cdot N^{\text {busy }} P_{\mathrm{m}}$ is penalty part. This means that when CRN senses correctly an idle subchannels, he would be rewarded and when CRN senses wrongly a busy subchannel, he would be penalized. $\omega_{\text {su }}$ and $\omega_{\text {pu }}$ represent the importance of CRN and PRN, respectively.

Figure 16 shows $U$ versus $P_{\mathrm{f}}$ with different SNR. It is seen that for a given SNR, there exists an optimal $P_{\mathrm{f}}$ such that $U$ is maximal. When SNR is at a low level, the CRN has to set a higher $P_{\mathrm{f}}$ to ensure $P_{\mathrm{m}}$ is low enough, whereas if SNR is high, CRN can select a lower $P_{\mathrm{f}}$ to obtain more transmission opportunities.

\section{Conclusion}

In this article, we develop an adaptive resources allocation scheme for CRNs in multiple PRNs environment. An MINP formulation is established to describe the scenario where a CRN exists around multiple PRNs. To obtain 
an adaptive resources allocations algorithm with polynomial time complexity, we design a hybrid objective function and two penalty functions, transforming the MINP into two unconstrained optimizations. Then, a PSO-based heuristic algorithm with low complexity initial topology is presented. Three computer simulations are carried out to verify the performance of our proposal. The results reflects that the proposed algorithm is efficient in terms of convergence and quality. Through comparisons, we show that the proposed algorithm outperforms traditional ARA algorithm both in sum capacity and proportional fairness.

\section{Competing interests}

The authors declare that they have no competing interests.

\section{Acknowledgements}

This study was supported by the National Basic Research Program of China (973 Program) (Grant No. 2009CB320402), and the National Natural Science Foundation of China (Grant No. 61032003 and 61001092).

Received: 24 October 2011 Accepted: 11 July 2012

Published: 12 August 2012

\section{References}

1. J Mitola, GQ Maguire, Cognitive radio: making software radios more personal. IEEE Personal Commun. Mag. 6(4), 13-18 (1999)

2. TYucek, $H$ Arslan, A survey of spectrum sensing algorithms for cognitive radio applications. IEEE Commun. Surv. Tutor. 11(1), 116-130 (2009)

3. E Axell, G Leus, E Larsson, Overview of spectrum sensing for cognitive radio. in Proceeding of International Workshop on 2nd Cognitive Information Processing (CIP) (Elba, Italy, 2010), pp. 322-327

4. X Kang, Y-C Liang, H Garg, L Zhang, Sensing-based spectrum sharing in cognitive radio networks. in Proceeding of IEEE Conference on Global Telecommunications Conference (GLOBECOM) (LA, USA, 2008), pp. 1-5

5. R Wang, V Lau, L Lv, B Chen, Joint cross-layer scheduling and spectrum sensing for ofdma cognitive radio systems. IEEE Trans. Wirel. Commun. 8(5), 2410-2416 (2009)

6. M-V Nguyen, HS Lee, Effective scheduling in infrastructure-based cognitive radio networks. IEEE Trans. Mobile Comput. 10(6), 853-867 (2011)

7. K Cumanan, R Krishna, Z Xiong, S Lambotharan, Multiuser spatial multiplexing techniques with constraints on interference temperature for cognitive radio networks. IET Signal Process. 4(6), 666-672 (2010)

8. $Y$ Zhang, C Leung, Resource allocation for non-real-time services in ofdm-based cognitive radio systems. IEEE Commun. Lett. 13(1), 16-18 (2009)

9. $Y$ Zhang, $C$ Leung, Resource allocation in an ofdm-based cognitive radio system. IEEE Trans. Commun. 57(7), 1928-1931 (2009)

10. Y Choi, H Kim, S wook Han, Y Han, Joint resource allocation for parallel multi-radio access in heterogeneous wireless networks. IEEE Trans. Wirel. Commun. 9(11), 3324-3329 (2010)

11. Z Shen, J Andrews, B Evans, Optimal power allocation in multiuser OFDM systems. in Proceeding of IEEE Conference on Global Telecommunications Conference (GLOBECOM), vol. 1 (San Francisco, USA, 2003), pp. 337-341

12. J Jang, KB Lee, Transmit power adaptation for multiuser ofdm systems. IEEE J. Sel. Areas Commun. 21(2), 171-178 (2003)

13. CY Wong, R Cheng, K Lataief, R Murch, Multiuser ofdm with adaptive subcarrier, bit, and power allocation. IEEE J. Sel. Areas Commun. 17(10), 1747-1758 (1999)

14. Z Shen, J Andrews, B Evans, Adaptive resource allocation in multiuser ofdm systems with proportional rate constraints. IEEE Trans. Wirel. Commun. 4(6), 2726-2737 (2005)

15. X Dong, J Wang, Y Zhang, M Song, R Feng, End-to-end qos provisioning in future cognitive heterogeneous networks. in Proceeding of IEEE Conference on Communications Technology and Applications (ICCTA) (Beijing, China, 2009), pp. 425-429
16. D Kumar, S Mahalaxmi, J Kumar, R Ramya, Adaptive resource allocation for real-time services in ofdma based cognitive radio systems. in Kaleidoscope: Beyond the Internet-Innovations for Future Networks and Services ITU-T (Pune, India, 2010), pp. 1-5

17. T Clancy, Achievable capacity under the interference temperature model. in Proceeding of IEEE Conference on 26th Computer Communications (INFOCOM) (Alaska, USA, 2007), pp. 794-802

18. IF Akyildiz, W-Y Lee, MC Vuran, S Mohanty, Next generation/dynamic spectrum access/cognitive radio wireless networks: a survey. 50(13), 2127-2159 (2006). [Online]. Available at http://www.sciencedirect.com/ science/article/pii/S1389128606001009

19. WJ Tang, QH Wu, Biologically inspired optimization: a review. Trans. Inst. Meas. Control. 31(6), 495-515 (2009)

20. T Renk, C Kloeck, D Burgkhardt, FK Jondral, D Grandblaise, S Gault, JC Dunat, Bio-inspired algorithms for dynamic resource allocation in cognitive wireless networks. in Proceeding of International Conference on 2nd Cognitive Radio Oriented Wireless Networks and Communications (CrownCom) (Orlando, USA, 2007), pp. 351-356

21. C Rieser, T Rondeau, C Bostian, T Gallagher, Cognitive radio testbed: further details and testing of a distributed genetic algorithm based cognitive engine for programmable radios. in Proceeding of IEEE Conference on Military Communications (MILCOM), vol. 3 (Monterey, Canada, 2004), pp. 1437-1443

22. D Maldonado, B Le, A Hugine, T Rondeau, C Bostian, Cognitive radio applications to dynamic spectrum allocation: a discussion and an illustrative example. in Proceeding of IEEE Symposium on New Frontiers in Dynamic Spectrum Access Networks (DySPAN) (Maryland, USA, 2005), pp. $597-600$

23. TR Newman, R Rajbanshi, AM Wyglinski, JB Evans, GJ Minden, Population adaptation for genetic algorithm-based cognitive radios. in Proceeding of International Conference on 2nd Cognitive Radio Oriented Wireless Networks and Communications (CrownCom) (Orlando, USA, 2007), pp. 279-284

24. Z Zhao, Z Peng, S Zheng, J Shang, Cognitive radio spectrum allocation using evolutionary algorithms. IEEE Trans. Wirel. Commun. 8(9), 4421-4425 (2009)

25. J-C Chen, C-K Wen, A novel cognitive radio adaptation for wireless multicarrier systems. IEEE Commun. Lett. 14(7), 629-631 (2010)

26. S Udgata, K Kumar, S Sabat, Swarm intelligence based resource allocation algorithm for cognitive radio network. in Proceeding of International Conference on 1st Parallel Distributed and Grid Computing (PDGC) (Solan (HP), India, 2010), pp. 324-329

27. J Perez-Romero, O Salient, R Agusti, L Giupponi, A novel on-demand cognitive pilot channel enabling dynamic spectrum allocation. in Proceeding of IEEE Symposium on New Frontiers in Dynamic Spectrum Access Networks (DySPAN) (Dublin, Ireland, 2007), pp. 46-54

28. RG Lyons, Understanding Digital Signal Processing, 2nd edn. (China Machine Press, China, 2005)

29. R Fletcher, An exact penalty function for nonlinear programming with inequalities. Math. Program. 5, 129-150 (1973). 10.1007/BF01580117. [Online]. Available at http://dx.doi.org/10.1007/BF01580117

30. J-M Yang, Y-P Chen, J-T Horng, C-Y Kao, Applying family competition to evolution strategies for constrained optimization. in Evolutionary Programming VI, ser. Lecture Notes in Computer Science, vol. 1213, ed. by P Angeline, R Reynolds, J McDonnell, and R Eberhart (Springer, Berlin, 1997), pp. 201-211. 10.1007/BFb0014812. [Online]. Available at http://dx. doi.org/10.1007/BFb0014812

31. J Kennedy, R Eberhart, Particle swarm optimization. in Proceeding of IEEE Conference on Neural Networks, vol. 4 (Nedlands, Western Australia, 1995), pp. 1942-1948

32. A Khosla, S Kumar, K Ghosh, A comparison of computational efforts between particle swarm optimization and genetic algorithm for identification of fuzzy models. in Fuzzy Information Processing Society (NAFIPS) (California, USA, 2007), pp. 245-250

33. E Laskari, K Parsopoulos, M Vrahatis, Particle swarm optimization for integer programming. in Proceeding of the Congress on Evolutionary Computation (CEC), vol. 2 (Hawaii, USA, 2002), pp. 1582-1587

34. M Clerc, J Kennedy, The particle swarm-explosion, stability, and convergence in a multidimensional complex space. IEEE Trans. Evol. Comput. 6(1), 58-73 (2002)

35. S Buljore, H Harada, S Filin, P Houze, KTsagkaris, O Holland, K Nolte, T Farnham, V Ivanov, Architecture and enablers for optimized radio 
resource usage in heterogeneous wireless access networks: the IEEE 1900.4 working group. IEEE Commun. Mag. 47(1), 122-129 (2009)

36. R Prasad, P Pawelczak, J Hoffmeyer, $\mathrm{H}$ Berger, Cognitive functionality in next generation wireless networks: standardization efforts. IEEE Commun. Mag. 46(4), 72-78 (2008)

37. D Gomez-Barquero, A Bria, J Monserrat, N Cardona, Minimal cost planning of dvb-h networks on existing wireless infrastructure. in Proceeding of IEEE Symposium on Personal, Indoor and Mobile Radio Communications (PIMRC) (Helsinki, Finland, 2006), pp. 1-5

38. R Tandra, A Sahai, SNR walls for signal detection. IEEE J. Sel. Areas Commun. 2(1), 4-17 (2008)

doi:10.1186/1687-1499-2012-252

Cite this article as: Ye et al:: Adaptive resource allocation for cognitive radio networks with multiple primary networks. EURASIP Journal on Wireless Communications and Networking 2012 2012:252.

\section{Submit your manuscript to a SpringerOpen ${ }^{\circ}$ journal and benefit from:}

- Convenient online submission

Rigorous peer review

- Immediate publication on acceptance

- Open access: articles freely available online

- High visibility within the field

- Retaining the copyright to your article

Submit your next manuscript at $\boldsymbol{\wedge}$ springeropen.com 